Addition of soluble fiber in low fat purified diets improves gut and metabolic health compared to traditional AIN diets

Laura Griffin $^{\mathrm{a}}$, Sridhar Radhakrishnan ${ }^{\mathrm{b}}$ and Michael Pellizzon ${ }^{\mathrm{b} *}$

${ }^{a}$ Givaudan, 245 Merry Lane, East Hanover, NJ 07936, USA

${ }^{\text {b} R e s e a r c h ~ D i e t s, ~ I n c . ~} 20$ Jules Lane, New Brunswick, NJ 08901, USA

\title{
Corresponding author*:
}

Michael Pellizzon, Research Diets, Inc. 20 Jules Lane New Brunswick, NJ 08901 USA.

E: michael.pellizzon@,researchdiets.com Ph: 732-317-5246

\section{List of abbreviations:}

1. Purified diet (PD)

2. Grain-based (GB)

3. AIN-76A rodent diet (76A)

4. AIN-93G rodent diet $(93 \mathrm{G})$

5. Cellulose (CEL)

6. Inulin (IN)

7. Fructo-oligosaccharides (FOS)

8. Open Standard Diet (OSD)

9. Enzyme Commission (EC)

10. Oral glucose tolerance test (OGTT)

11. Permutational Analysis of Variance (PERMANOVA)

12. Analysis of Variance (ANOVA) 


\section{Conflict of Interest and Funding Disclosure:}

Research Diets, Inc. provided the funding for this research work.

SR and MP are employees of Research Diets Inc., the manufacturer of the purified ingredient diets utilized in these studies.

\section{Keywords:}

1. Purified diets

2. Grain-based diets (chow)

3. Sucrose

4. Dextrose

5. Fiber

6. Fructo-oligosaccharides

7. Inulin

8. Cellulose

9. Glucose tolerance

10. Gut Microbiota. 


\section{ABSTRACT}

2 Background: Purified diets (PDs) contain refined ingredients with one main nutrient, allowing

3 for greater control relative to grain-based diets (GBDs), which contain unrefined grains and

4 animal byproducts. Traditional PDs like the AIN-76A (76A) and AIN-93G (93G) can negatively

5 impact metabolic and gut health when fed long-term, in part due to lower total fiber, no soluble

6 fiber, and higher sucrose content.

7 Objective: Two studies were conducted to determine how PDs with reduced sucrose and

8 increased fiber (soluble and insoluble) influence metabolic and gut health in mice compared to

9 traditional AIN PDs or GBDs.

10 Methods: In study 1, C57Bl/6N mice consumed a GBD (5002), 76A, 93G, or 2 Open standard

11 PDs (OSDs) with reduced sucrose and higher fiber for 88 days. Body composition and metabolic

12 parameters were assessed. In study 2, C57B1/6N mice consumed either 2 GBDs (5001 or 5002)

13 or OSDs with different types/levels of fiber for 14 days. Microbiome alterations and predicted

14 functional metagenomic changes were measured.

15 Results: OSD marginally influenced body weight and adiposity, but improved glucose tolerance

16 relative to $93 \mathrm{G}(\mathrm{p}=0.0131)$ and $76 \mathrm{~A}(\mathrm{p}=0.0014)$. Cecal and colonic weights were lower in

17 mice fed cellulose-based PDs compared to those fed GBDs and soluble fiber PDs. Soluble fiber

18 diets reduced alpha diversity and showed similar beta diversity, which differed from cellulose

19 fed PDs and GBDs. Certain genera associated with improved gut health such as Bifidobacteria

20 and Akkermansia were significantly elevated by soluble fibers PDs ( $\mathrm{p} \leq 0.01)$. Some metabolic

21 pathways related to carbohydrate and fatty acid metabolism were affected by PDs. 
bioRxiv preprint doi: https://doi.org/10.1101/2022.02.28.482306; this version posted March 2, 2022. The copyright holder for this preprint (which was not certified by peer review) is the author/funder, who has granted bioRxiv a license to display the preprint in perpetuity. It is made available under aCC-BY-ND 4.0 International license.

22 Conclusion: PDs formulated with lower sucrose and increased fiber content, particularly soluble

23 fiber, blunted elevations in metabolic parameters and favorably impacted microbiota and

24 metagenome in $\mathrm{C} 57 \mathrm{BL} / 6 \mathrm{~N}$ mice. 


\section{INTRODUCTION}

26 Of the many environmental variables that affect the phenotype of an animal, diet is one that can

27 be easily controlled. Laboratory rodent diets are classified into two main types: grain-based diets

28 (GBDs) or purified diets (PDs). GBDs (or cereal based diets or natural ingredient diets) are

29 typically closed formulas and made with grain-based ingredients and animal byproducts (1).

30 While they provide nutrition for growth and overall health, they contain non-nutritive ingredients

31 such as phytochemicals and potential toxins such as endotoxins, mycotoxins and heavy metals

32 from several ingredients, which may vary from batch-to-batch and potentially influence

33 phenotype (1-3).

35 PDs are 'open' formulas made with defined concentrations of ingredients that are highly refined,

36 each providing one main nutrient (i.e., sucrose is mainly carbohydrate, corn oil is mainly fat and

37 casein is mainly protein). Being highly refined, the non-nutrient content is minimal and the

38 nutrient compositions of both macro- and micronutrients in PDs are well defined, limiting the

39 variability from batch-to-batch $(1,4)$. Since each nutrient/ingredient is added individually, it also

40 allows the researcher to selectively manipulate nutrients, thus providing a wide range of

41 modifications (for e.g. high-fat/protein, high fructose, ketogenic) to study different phenotypes in

42 rodents and in other animal models. The AIN-76A and AIN-93G diets $(5,6)$ are two of the most

43 commonly used PDs, which can provide adequate growth and health of rats and mice; however,

44 there have been reports $(7,8)$ of mild metabolic dysfunction (increased body weight, body fat,

45 mild insulin resistance, hyperlipidemia, etc.) in animals consuming these diets, relative to GBD

46 fed animals. While several differences exist between these two types of diets, these perturbations

47 may be in part due to certain ingredients in these diets, including their higher sucrose content 
$48(10 \%$ and $50 \% \mathrm{w} / \mathrm{w}$ in $93 \mathrm{G}$ and $76 \mathrm{~A}$, respectively) and a low amount of total and mostly non-

49 fermentable fiber $(5 \%$ cellulose $)(9)$. This is in stark contrast to the presence of minimal amounts

50 of sucrose and relatively higher amounts $(15-25 \% \mathrm{w} / \mathrm{w})$ of fiber in GBDs. In addition, GBDs

51 also contain diverse sources of fiber including soluble (beta-glucan, pectin, etc.), partially soluble

52 (hemicellulose) and insoluble fibers (celluloses, lignin, etc.) $(1,2)$.

54 Metabolic differences that are observed in rodents consuming AIN PDs relative to those fed

55 GBDs may be driven in part by differences in sucrose and fiber, in particular, soluble fiber.

56 Modifications to these PDs include replacement of sucrose with sources such as corn starch and

57 dextrose to minimize fructose, an initiator of metabolic disease, including insulin resistance,

58 glucose intolerance, and hyperlipidemia(11,12). Even relatively low levels of sucrose may

59 influence glucose tolerance over more chronic feeding periods(13). The fiber content of PDs can

60 also be increased and furthermore, refined soluble fiber sources, such as fructo-oligosaccharides

61 (FOS) or inulin, which have been long known to promote metabolic health via the gut (14-16),

62 can be added to these diets. We and others have shown that the addition of soluble fibers such as

63 inulin, in the context of a diet higher in fat can reduce body weight, adiposity, and blood and

64 liver lipids, inflammation, and improve glucose tolerance of rodents, perhaps in part, due to

65 elevated short-chain fatty acid production by the gut bacteria or other factors (17-19).

66

67 The purpose of this study, therefore, was to assess whether changes to the carbohydrate and fiber

68 components of the AIN PDs could improve metabolic health of rodents. Furthermore, we

69 determined how changes in the type and amount of fiber affected gut health/microbiome profile.

70 Metabolic and gut microbiome effects in mice fed PDs were also compared to those fed GBDs. 


\section{METHODS}

\section{Dietary Formulations.}

73 Study 1: In addition to the traditional AIN PDs, AIN-76A (76A) and AIN-93G (93G), we used

74 two modified versions of the AIN diets, referred to as the Open Standard Diets (OSDs). The

75 nutritional profiles of the four PDs utilized are presented in Table 1. The OSDs contained only

76 trace levels of sucrose (in the vitamin and mineral mixes), providing around $1 \%$ of total kcals.

77 The diet D11112201 (OSD) was formulated with $100 \mathrm{gm}$ of added fiber per $4084 \mathrm{kcals}$ in a 3:1

78 ratio of cellulose to inulin (75 g cellulose and $25 \mathrm{~g}$ inulin per $4084 \mathrm{kcals}, 9.3 \%$ fiber w/w), with

79 inulin providing approximately $1.5 \mathrm{kcal} / \mathrm{g}$ from fermentation (20). D11112202 (OSD + F)

$80(20.5 \%$ total fiber w/w) contained three times as much cellulose, $(225 \mathrm{~g}$ per $4084 \mathrm{kcals})$ as

81 D11112201, but the same amount of inulin (25 g per 4084 kcals), to be more in line with GBDs

82 which contain higher amounts of fiber as insoluble fiber with some soluble fiber. All PDs were

83 formulated by Research Diets, Inc. (New Brunswick, NJ, USA). These diets were compared to

84 the GBD, LabDiet 5002 (chow) (St. Louis, MO). Fiber content of this chow was analyzed by the

85 Laboratory of Dr. Kelly Swanson, Dept. of Animal Sciences, University of Illinois. The total

86 fiber content was $23.9 \%$. Insoluble fiber content was $18.6 \%$ and soluble fiber content was $5.3 \%$.

87 Study 2: To further understand the role of fiber type and concentration in PDs and how they

88 compare to GBDs, we used six additional versions of the OSD with either 100 or $200 \mathrm{~g}$ of

89 cellulose (CEL), inulin (IN), or fructo-oligosaccharides (FOS) per 4084 kcals. The six

90 experimental OSDs (100CEL, 200CEL, 100IN, 200IN, 100FOS, and 200FOS) were compared to

91 two GBDs, LabDiet 5001 and 5002 (LabDiet, St. Louis, MO), see Table 2. Fiber content of 5001

92 and 5002 were analyzed by Covance Laboratories (Madison, WI); 5001: 18.7\% total, 15.9\%

93 insoluble, $2.8 \%$ soluble, 5002: $18.2 \%$ total, 14.9\% insoluble fiber, and 3.3\% soluble fiber. 


\section{Animals and Study Design.}

95 Study 1: This study was conducted at MuriGenics, Inc. (Vallejo, CA, USA). Weanling male

96 C57Bl/6N mice $(n=75)$ were purchased from Charles River Laboratories. Mice were housed $(n$

$97=5 /$ cage on Alpha-Dri bedding) in micro isolators on a $12 \mathrm{hr}$ light/dark cycle and maintained on

98 chow (LabDiet 5001). Food and water were provided ad libitum. At 4 weeks of age, mouse cages

99 were randomly assigned to one of the five treatment groups ( 3 cages/treatment; $n=15$

100 animals/treatment). Mice were maintained on the experimental diets for 88 days. Food and water

101 intake per cage and body weights were measured weekly throughout the study.

102 Study 2: The study was conducted at Charles River Laboratories (Wilmington, MA, USA). Male

103 weanling C57Bl/6N mice $(\mathrm{n}=54)$ from Charles River Laboratories were housed 3 per cage on

104 Alpha-Dri bedding. Mice were fed 5001 ad libitum and housed in standard vivarium conditions

105 on a 12-hr light/dark cycle. After 2 days following receipt, six mice were sacrificed for baseline

106 data. At 4 weeks of age, initial body weight measurements were collected and mouse cages were

107 randomly assigned to one of 8 treatment groups ( 2 cages/treatment, $n=6 /$ treatment $)$. Animals

108 were maintained on the experimental diets for 14 days. Body weights were measured prior to

109 euthanasia.

111 Glucose Tolerance Test.

112 Study 1: On day 83, an oral glucose tolerance test (OGTT) was performed after 12 weeks on the

113 experimental diets with modification, as described previously (21). Briefly, following a 6-hour

114 fast, baseline blood glucose measurements were taken. Immediately afterwards, mice were

115 gavaged a $20 \%$ dextrose solution to deliver 2 g glucose $/ \mathrm{kg}$ body weight. Blood glucose 
116 measurements were collected via the tail vein at 30 minute intervals over the course of 2 hours.

117 Food was restored upon completion of the procedure.

119 Euthanasia and Necropsy.

120 Study 1: On day 88, mice were fasted for 6 hours, blood samples were collected via cardiac

121 puncture, and euthanized. Serum was separated and stored at -80C until analysis. Following

122 blood collection, mice were perfused with saline. Carcass, liver, and select adipose deposits

123 (mesenteric, gonadal, inguinal, retroperitoneal) were weighed. Livers were flash frozen in liquid

124 nitrogen and stored at -80C for triglyceride analysis.

125 Study 2: At the end of 14 days, animals were euthanized using CO2. Following CO2

126 asphyxiation, the cecum and colon were harvested together with their contents remaining intact.

127 The total tissue was weighed. For the first animal in each group, an image of the colon and

128 cecum (attached) was taken next to a standard ruler. The colon and cecum were separated from

129 each other (contents still intact) and each were weighed. Colon and cecum contents were

130 collected into separate vials and placed on dry ice before being stored at $-70^{\circ} \mathrm{C}$. The colon and

131 cecum were cleaned with DD water, blotted dry, and weighed individually. Colonic length was

132 recorded once the tissue was cleaned and dry.

134 Serum and Triglyceride Analysis.

135 Study 1: Serum from each animal was analyzed for fasting blood glucose, leptin, triglycerides,

136 and total cholesterol at MuriGenics (Vallejo, CA, USA). Insulin and leptin were analyzed using a

137 multiplex assay. Liver samples were assayed for triglyceride content by Vascular Strategies LLC

138 (Wynnewood, PA, USA). 


\section{Microbiome Analysis.}

140 Sequencing QC and Analysis: 16S rRNA sequencing was performed on the cecum and colon

141 content samples at the Argonne National Laboratory (Argonne, IL, USA). Briefly, total DNA

142 was extracted from the samples, and the V3-V4 regions of the 16S rRNA were amplified using

143 PCR and sequenced using their MG-RAST pipeline. Sequence data from this pipeline was

144 transferred to Diversigen (Minneapolis, MN, USA) for subsequent analysis. Raw FASTQs for all

145 samples were run through the QC pipeline at Diversigen. Briefly, this involves trimming of

146 adapter sequences (if present) using the program cutadapt followed by filtering of reads to

147 remove any with a mean Q-score of $<30$. Next, FASTQ files were run through their standard

148 dada2 pipeline to produce the raw Amplicon Sequence Variant (ASV) count table. The raw table

149 was then filtered to remove any ASVs at $<0.0001 \%$ sum relative abundance across all samples.

150 Finally, we used the filtered ASV table as input to PICRUSt2 infer functional metagenomic

151 content of all samples. The outputs of this were an abundance table of predicted Enzyme 1

152 Commission (EC) numbers, as well as an abundance table of predicted Metacyc Pathways

153 derived from the EC abundance data.

155 Taxa and Functional Summary Plots: The filtered ASV table was used to generate summary

156 stacked bar plots of relative abundances per-sample at the phylum and ASV levels for both

157 cecums and colons. Comparisons between phyla and genera by diet and tissue type can be found

158 in the supplemental tables. For visualization purposes, only the top 20 most abundant genera are

159 plotted in the Genus-level plot, with the rest allocated to an "Other" category. At the Phylum

160 dlevel, the ratio of Firmicutes/Bacteroidota was calculated per-sample by dividing the relative

161 abundances for each of these. The results of these ratios were then plotted across all diet groups. 
162 To assess whether the Firmicutes/Bacteroidota ratio differed across diet groups, we used a one-

163 way ANOVA across treatment groups followed by Tukey's HSD. To examine broad patterns of

164 predicted functional genomic content across diet groups, the predicted EC count table was used

165 as an input. EC numbers in this table were then grouped according to six broad functional

166 categories as defined in the Kyoto Encyclopedia of Genes and Genomes (KEGG) database: (1)

167 Carbohydrate Degradation and Absorption, (2) Fructose and Mannose Metabolism, (3) Galactose

168 Metabolism, (4) Starch and Sucrose Metabolism, (5) Fatty Acid Metabolism and (6) Fatty Acid

169 Biosynthesis. Counts for ECs found within each of these categories were then aggregated per-

170 category, and the resulting count data was plotted as boxplots. To test whether counts differed

171 across diet groups within these categories, we used a one-way ANOVA across all treatment

172 groups followed by Tukey’s HSD.

173

174 Alpha and Beta Diversity Analyses: Using the ASV, EC and Pathways tables, we rarefied each

175 table to the sample with the lowest mapped counts. We then calculated three alpha diversity

176 metrics for each of the three feature tables: the Shannon index, the Chao1 index and Observed

177 Features. To examine all pairwise comparisons of each treatment group to every other group, we

178 used a one-way ANOVA across all treatment groups followed by Tukey's HSD. All results were

179 then plotted as box plots with strip charts overlaid to show all data points. To assess differences

180 in between-sample (i.e. beta) diversity, we calculated distance matrices for all three rarefied

181 feature tables using the Bray-Curtis Dissimilarity metric. Next, we used Permutational

182 Multivariate Analysis of Variance (PERMANOVA) from the R package vegan to assess

183 differences in beta diversity between treatment groups. The results were then plotted using

184 principal coordinates analysis (PCoA) plots with samples colored by treatment group. We also 
185 determined the beta diversity variability within each treatment group by calculating the distance-

186 to-centroid for every sample within its group. We then used the same statistical methodologies

187 used for alpha diversity to determine if beta diversity variability is different between any

188 treatment groups. All results of statistical testing are included with individual plots.

190 Differential Abundance Analysis: Finally, we assessed whether any taxa - Phylum or Genus

191 level or functions (Metacyc Pathways-level) differ in abundance between the treatment groups in

192 the study. Due to the compositional nature of microbiome abundance data, we utilized the $\mathrm{R}$

193 package $A L D E x 2$. Briefly, this package performs differential abundance testing of count data

194 across samples between two experimental groups by starting with zero-estimation for any

195 features with zero abundance in some samples but not others. Next, per-feature technical

196 variation for each feature is estimated for each sample using Monte-Carlo sampling from a

197 Dirichlet distribution. Each instance of Monte-Carlo sampling is then transformed using the

198 Centered LogRatio (CLR) transformation, at which point pairwise statistical testing is performed

199 between experimental groups using the CLR-transformed abundance values. This process is

200 repeated for each instance, and results of statistical testing are aggregated yielding adjusted p-

201 values (Benjamini-Hochberg corrected) for each feature in the differential abundance test of

202 interest. We used the above procedure to test for differential abundance between every pairwise

203 comparison of treatment groups. Individual $\mathrm{p}$-value tables of the results of statistical testing for

204 every feature in a given pairwise comparison is presented as supplementary files. For all

205 statistical tests, an adjusted $p$-value of $<0.05$ was used as a threshold for significance. 
bioRxiv preprint doi: https://doi.org/10.1101/2022 02 28.482306; this version posted March 2, 2022. The copyright holder for this preprint (which was not certified by peer review) is the author/funder, who has granted bioRxiv a license to display the preprint in perpetuity. It is made available under aCC-BY-ND 4.0 International license.

\section{Statistical Analysis.}

209 For study 1, all data were analyzed by 1-way ANOVA with GraphPad Prism. Post-hoc analyses

210 were performed if $\mathrm{p}<0.05$ using Tukey's HSD for comparisons among groups (both studies).

211 For study 2, to examine all pairwise comparisons between treatment groups, 2-way ANOVA

212 followed by Tukey's HSD was employed using R. Microbial statistics are presented in the

213 section above. An adjusted $\mathrm{p}$-value of $\mathrm{p}<0.05$ was used as a threshold for significance for all

214 statistical tests.

215

216

217

218

219

220

221

222

223

224

225

226

227

228

229

230 


\section{RESULTS}

232 STUDY 1: Body Composition and Metabolic Assessment: Over time, the average body

233 weights of OSD and 93G treatment groups were slightly higher (by mean of $2.3 \mathrm{~g}$ ) compared

234 with those fed the OSD+F, 76A, and 5002 diets (Figure 1). Terminal body weights were similar

235 for all groups, though OSD was statistically higher than 76A (p = 0.0102), but similar to $5002(\mathrm{p}$

$236=0.163)$ and $93 \mathrm{G}(\mathrm{p}=0.8223)$ and additional fiber as cellulose in the OSD $+\mathrm{F}$ group blunted this

237 effect. All the individual fat pad (mesenteric, gonadal, retroperitoneal and inguinal) weights

238 were generally similar among groups. OSD fed mice had significantly heavier gonadal fat pads

$239(\mathrm{p}=0.0312)$ and total fat $(\mathrm{p}=0.0479)$ compared to 5002 and significantly heavier inguinal

240 (subcutaneous) fat pads $(\mathrm{p}=0.0036)$ compared to 93G (Table 3); this difference was blunted by

241 the addition of extra cellulose in the OSD+F group. The carcass weights were generally similar

242 across groups, although in the OSD group, the carcass weight was significantly higher than in the

24376 Aroup $(\mathrm{p}=0.019)$. Adiposity index ( $\mathrm{g}$ total fat / $\mathrm{g}$ carcass) was also similar among groups

244 though it tended to be higher in OSD than $5002(\mathrm{p}=0.0548)$, but significantly greater in OSD vs.

$24593 \mathrm{G}(\mathrm{p}=0.0372)$ due mainly to a lower inguinal fat pad weight in the latter group. The addition

246 of cellulose in OSD $+\mathrm{F}$ blunted the adiposity index and resulted in similar levels relative to all

247 groups (Table 3). The liver weights were also not significantly different among the five groups

248 (data not shown).

250 Serum Biochemistry: Glucose homeostasis was assessed prior to study termination with an oral

251 glucose tolerance test (Figure 2). All groups had similar 6-hr fasting blood glucose levels

252 (Figure 2A). Glucose tolerance was significantly reduced in 93G and 76A mice (as shown by a

253 greater area under the curve) compared to both chow (vs. 93G, $\mathrm{p}=0.0399$, vs. 76A, $\mathrm{p}=0.005$ ) 
and OSD mice (vs. 93G, $\mathrm{p}=0.0131$; vs. 76A, $\mathrm{p}=0.0014)$ (Figure 2B). OSD+F was

255 intermediate and similar to all groups. Additional measurements of 6-hr fasting serum glucose

256 and insulin measurements were made at study termination (Table 4). Serum cholesterol was

257 significantly higher in all PD groups compared to chow (OSD, $\mathrm{p}=0.0037$; OSD+Fiber, $\mathrm{p}=0.0005$;

$25876 \mathrm{~A}, \mathrm{p}=0.001)$ and was highest in the 93G group $(\mathrm{p}<0.0001)$ (Table 4). Serum triglycerides

259 were similar among groups, but were significantly lower for 76A compared to chow $(\mathrm{p}=$

260 0.0009). Serum leptin was higher in OSD and 93G fed mice compared to those fed OSD+F

261 (OSD, $\mathrm{p}=0.0207 ; 93 \mathrm{G}, \mathrm{p}=0.0057)$ and chow (OSD, $\mathrm{p}=0.007 ; 93 \mathrm{G}, \mathrm{p}=0.0017)$, while 76A had a

262 similar leptin level relative to all groups. Liver triglycerides were not different between chow,

$263 \mathrm{OSD}+\mathrm{F}$ and 76A fed mice, but OSD and 93G had higher levels compared to chow (OSD,

$264 \mathrm{p}=0.0008 ; 93 \mathrm{G}, \mathrm{p}=0.0002)$ and 93G had higher levels than 76A $(\mathrm{p}=0.0328)$.

265

STUDY 2: Morphological Changes After 14 Days on High-Fiber Purified Diets.

267 The body weight of animals in all groups were similar at the end of the two week experimental

268 period. Body weight and weight gain in only the 200FOS group were significantly lower

269 compared to the GBD groups (5001, $\mathrm{p}=0.0498 ; 5002, \mathrm{p}=0.0191)$ (Table 5). Despite minimal

270 differences in weight gain, rapid changes to lower intestinal morphology were observed after two

271 weeks on certain diets. Representative pictures of cecums and colons from each group indicate

272 that regardless of fiber amount, the cellulose supplemented PDs yielded smaller cecums and

273 shorter colons compared to soluble fiber supplemented PDs or the GBDs (Figure 3). As

274 suggested by the differences in the photos, statistical analysis of the organ weights and lengths

275 showed similar trends and significant differences between groups (Table 5). Significantly shorter

276 colons were observed in the 100CEL $(\mathrm{p}=0.0045)$ and 100FOS $(\mathrm{p}=0.0123)$ groups compared to 
277 the 5002 group, but both PD groups were similar to 5001; however, all other PD groups were

278 statistically similar to both GBD groups (5001 and 5002). Cecum plus colon wall weights for the

$279200 \mathrm{IN}$ and 200FOS groups were similar to one another but significantly elevated compared to all

280 other treatments (200IN and 200FOS vs. most groups, $\mathrm{p}<0.0001$; FOS200 vs. 5002, $\mathrm{p}=0.0008$ );

$281200 \mathrm{IN}$ and 200FOS were similar to one another. 100CEL had significantly lower cecum plus

282 colon weights than both GBDs (vs. 5001, $\mathrm{p}=0.0253$; vs. 5002, $\mathrm{p}<0.0252$ ) and the two inulin

283 groups and 200FOS (100CEL vs. 100IN, p=0.0255; 100CEL vs. 200IN and 200FOS, p<0.0001),

284 while 200CEL increased it slightly and was similar to 100IN, 100FOS, and GBD groups. The

285 100IN group maintained similar cecum plus colon weights as GBDs. Cecum weights of the

286 different groups followed similar trends as cecum plus colon weights with 200IN and 200FOS

287 being similar to one another but higher compared to all other groups (200IN vs. all groups,

$288 \mathrm{p}<0.0001 ; 200 \mathrm{FOS}$ vs. 5001, $\mathrm{p}=0.0011 ; 200 \mathrm{FOS}$ vs. 5002, $\mathrm{p}=0.0006 ; 200 \mathrm{FOS}$ vs. 100CEL and

289 200CEL, $\mathrm{p}<0.0001 ; 200 \mathrm{FOS}$ vs. 100IN, $\mathrm{p}=0.0031 ; 200 \mathrm{FOS}$ vs. 100FOS, $\mathrm{p}=0.0009)$. Cecum

290 weight relative to BW in $100 \mathrm{IN}$ tended to be higher than those of 100CEL ( $p=0.074)$, but 200IN

291 had significantly higher cecum weights than 200CEL $(p<0.0001)$. The 100IN group allowed for

292 similar cecum weights as for those fed GBDs. Colon weights alone followed a similar trend and

293 were more varied. The 200IN group tended to have higher colon weights than those fed

294 200CEL, but not statistically higher $(\mathrm{p}=0.0533)$ and the addition of more inulin or FOS also

295 tended to increase colon weights. Notably the 100CEL group demonstrated significantly

296 reduced colon weight compared to the GBD groups $(5001, \mathrm{p}=0.048 ; 5002, \mathrm{p}=0.0186$. In

297 contrast, 200CEL maintained similar colonic weights as GBD groups. 


\section{Changes to the Prominent Microbial Taxa after 14 Days on the Dietary Treatments.}

\section{a. Alpha and Beta diversity measures}

302 To assess the response of the microbiome to the different dietary treatments, 16S rRNA

303 sequencing was performed on the cecum and colon contents of each animal. When examining

304 differential abundance for both taxa and functions across treatment groups, we found a large

305 number of features at all levels examined that differed between at least one (and usually more)

306 pairwise comparisons of treatment groups. In the cecum samples, 168 ASVs (50 Genera, 25

307 Families and 7 Phyla), 1342 Ecs, and 282 metacyc pathways were differentially expressed. In the

308 colon samples, it was 165 ASVs (51 Genera, 24 Families and 7 Phyla), 1322 Ecs, and 279

309 metacyc pathways. Globally, although the diets 5001 and 5002 have slightly different

310 composition, there were no noteworthy or significant changes to report between these two

311 groups. With respect to alpha diversity, the soluble fiber diets (IN and FOS) were similar and

312 both soluble fibers significantly reduced species richness compared to the GBDs and cellulose

313 based diets in cecums and colons for Chao1, Observed, and Shannon diversity indices (Figure

314 4). Dietary soluble fiber treatments significantly influenced alpha diversity metrics for both sites

315 ( $\mathrm{p}<0.001$ for all analyses). The GBDs were able to support the greatest number of species in

316 both tissue types regardless of the diversity metric used and in most cases, both the 100CEL and

317 200CEL groups were also able to maintain a statistically similar number of species as GBDs.

318 Overall, fiber dose did not have a significant impact on alpha diversity. In terms of beta

319 diversity, PCoA plots depicting the Bray-Curtis dissimilarity of ASVs for cecums and colons

320 (Figure 5) indicated that treatment groups were quite distinct from one another. In fact, three

321 significantly different clusters of microbial communities were observed in both cecums $(\mathrm{p}=$

$3220.001)$ and colons $(\mathrm{p}=0.001)$. Once again, dietary fiber types appeared to be the primary 
323 differentiating factor, with the three distinct clusters consisting of soluble fiber-based diets (both

324 IN and FOS clustered together), cellulose-based diets, or GBDs. Fiber dose did not appear to

325 influence beta diversity, as the low dose and high dose treatments for each fiber type were

326 clustered together in both sites of sampling.

\section{b. Phylum changes}

329 When looking more closely at the relative proportions of taxa, shifts in relative abundance of

330 certain microbes were observed at the phyla and genus level (Figure 6) and associated P values

331 for individual microbial taxa differences can be found in the Supplementary Tables 1-4. At the

332 phylum level, Firmicutes was the dominant phyla found in the GBD treatment groups, followed

333 by Bacteroidota for cecum samples (Figure 6A). The opposite trend was observed in the colon

334 samples, where Bacteroidota was the dominant phylum (Figure 6B). Interestingly, all of the

335 soluble fiber diets significantly reduced the Firmicutes/Bacteroidota ratio in the cecum samples

336 (Supplementary Figure 1A) compared to the GBDs; however, in the colon, it was not

337 significant at $\mathrm{P}=0.025$ (except for the 200IN group) (Supplementary Figure 1B). The addition

338 of cellulose to the diets led to minor phylum shifts, such as increased abundance of

339 Deferribacterota in the cecums. This shift was significant for 100CEL $(p=0.0226)$ and 200CEL

$340(p=0.0183)$ groups compared to 5002 (Supplementary Table 1). No differences were observed

341 between the cellulose diets and the GBDs at the phylum level in the colon samples. In contrast,

342 major phylum-level shifts were observed in both tissue types for the mice fed the soluble-fiber

343 diets. The soluble fiber diets generally reduced the abundance of Firmicutes in both cecum and

344 colon, reaching significance for the 200IN, 100IN, and 200FOS groups compared to both GBDs

345 ( $\mathrm{p}$ values $<0.039$ in each case for both tissue sites) (Supplementary Table 1 and 2). In the 
cecums, marked reductions in Firmicutes abundance alongside elevations in Verrucomicrobiota

were observed, particularly for the FOS treatment groups. In fact, Verrucomicrobiota abundance

was significantly higher for 100FOS compared to $5001(\mathrm{p}=0.0137)$, while the 200FOS group

349 demonstrated significantly lower Firmicutes abundance compared to $5001(\mathrm{p}=0.012)$ and 5002

$350(p=0.0176)($ Supplementary Table 1). Specifically in the colon, Verrucomicrobiota abundance

351 was generally elevated compared to the GBDs, reaching significance for 200FOS $(p=0.0133)$,

352 100FOS $(\mathrm{p}=0.0106)$ and $100 \mathrm{IN}(\mathrm{p}=0.0245)$ compared to 5001, and for 100FOS $(\mathrm{p}=0.0411)$

353 compared to 5002. Both FOS groups also demonstrated significant elevations in the phylum

354 Actinobacteriota compared to both GBDs (Supplementary Table 1). Similar trends were

355 observed in the colon samples. Colon Actinobacteriota populations were also significantly

356 increased by both FOS treatment groups compared to both GBDs ( $\mathrm{p}$ values $<0.026$ )

357 (Supplementary Table 2).

\section{c. Genus level changes}

360 Notable shifts at the genus level were also observed due to the different dietary treatments. In the

361 cecum samples (Figure 6C), prominent genera present in the GBDs included the

362 Lachnospiraceae NK4A136 group and an unclassified genus from the family Muribaculaceae.

363 Interestingly, Lachnospiraceae NK4A136 group was significantly higher in abundance for both

364 GBDs compared to the cellulose and FOS-based diets, and tended to be higher than 200IN (5001

365 vs. $200 \mathrm{IN}, \mathrm{p}=0.062$, 5002 vs. $200 \mathrm{IN}, \mathrm{p}=0.093)$, but not different from those fed 100IN (5001 vs.

$366100 \mathrm{IN}, \mathrm{p}=0.53 ; 5002$ vs. $100 \mathrm{IN}, \mathrm{p}=0.57$ ). The family Oscillospiraceae (undefined genus) was

367 significantly increased by cellulose groups compared to both GBDs and most of the soluble fiber

368 groups, except where 100CEL was not significantly different from 100IN (p=0.14). Alistipes 
was significantly elevated by 200CEL relative to what was found in both GBDs (vs. 5001, $\mathrm{p}=0.035$; vs. 5002, $\mathrm{p}=0.009)$ and compared to all soluble fiber groups $(\mathrm{p}<0.02)$. All of the soluble

371 fiber diets at both doses, relative to GBDs, significantly increased the relative abundance of

372 Bifidobacteria, Akkermansia, and Faecalibaculum (GBDs vs. all soluble fiber diets, $\mathrm{p} \leq 0.01$ ),

373 while decreasing Roseburia (GBDs vs. $200 \mathrm{IN}$ or $200 \mathrm{FOS}$ diets, $\mathrm{p}<0.01$; vs. $100 \mathrm{IN}$ or $100 \mathrm{FOS}$,

$374 \mathrm{p}<0.05$ ); similar trends were also observed relative to cellulose-based diets (Bifidobacteria,

375 Akkermansia, and Faecalibaculum, $\mathrm{p}=0.01$; for Roseburia, $\mathrm{p}<0.02$ ). Almost all the PDs caused a

376 significant increase in the relative abundance of genus Bacteroides $(\mathrm{p} \leq 0.03)$ except for 5002

377 relative to $100 \mathrm{CEL}(\mathrm{p}=0.13$ ) and all PDs had more of the family Tannerellaceae (genus

378 undefined) compared to GBDs ( $\mathrm{p} \leq 0.01)$ (Figure 6C, Supplementary Table 3). Bacteroides was

379 significantly increased by soluble fiber diets at lower fiber doses, but not at higher doses

380 compared to the cellulose diets at the same dose (100IN and 100FOS vs. 100CEL, $p=0.01$;

$381200 \mathrm{IN}$ and $200 \mathrm{FOS}$ vs. 200CEL, $\mathrm{p} \geq 0.09$ ). Dose of fiber (regardless of type of fiber) had no

382 significant influence on any of the above mentioned genera.

384 Similar trends in relative abundance at the genera level were observed in the colon samples

385 (Figure 6D). Not surprisingly, the soluble fiber based diets significantly increased the abundance 386 of Bifidobacteria, Akkermansia, and Faecalibaculum compared to cellulose and GBDs. Unlike in 387 the cecums, Roseburia abundance was significantly increased for 100IN ( $p=0.0194), 200 \mathrm{IN}(\mathrm{p}$ $388=0.0105)$, and 200FOS $(\mathrm{p}=0.011)$ relative to 5002; however, there were no differences

389 compared to the 5001 group for any PDs (Supplementary Table 6). Similar to the cecum 390 samples, all PD groups increased the relative abundance of the genera Bacteroides (except 391 100CEL vs. 5002, p=0.06) and Prevotella_UCG-001, and the Tannerellaceae family (undefined 
genus) compared to GBDs. The family Tannerellaceae (undefined genus) was also significantly

higher for 100CEL compared to $100 \mathrm{IN}(\mathrm{p}=0.02)$. Bacteroides was significantly increased by

394 100IN and 100FOS relative to 100CEL $(p=0.01)$ and for 200FOS relative to 200CEL $(p=0.045)$,

395 but not changed by fiber dose. In contrast, an undefined genus in the family Muribaculaceae was

396 significantly reduced by $100 \mathrm{IN}$ and $100 \mathrm{FOS}$ relative to 100CEL $(\mathrm{P} \leq 0.03)$ and $200 \mathrm{FOS}$ vs.

397 200CEL $(\mathrm{P}=0.012)$. Collectively, these differences clearly demonstrate that different fiber types

398 support the growth of different microbes present in the GI tract and that both soluble fibers

399 tended to support similar microbial growth.

\section{Predicted Metabolic Functions of Microbiota.}

402 The predicted function of the gut microbiota with respect to metabolism in response to the

403 dietary treatments was evaluated using the PICRUSt program. When examining the gene counts

404 for specific categories of metacyc pathways for cecum (Figure 7) and colon (Supplementary

405 Figure 2) samples, key differences between treatment groups were observed. These pathways

406 were related to carbohydrate digestion and absorption, fructose and mannose metabolism,

407 galactose metabolism, starch and sucrose metabolism, fatty acid metabolism, and fatty acid

408 biosynthesis (Figure 7). Generally speaking, the PDs had higher gene counts than GBDs for all

409 the six tested pathways in the cecal samples, but only the 200CEL and 200IN treatments

410 significantly elevated the gene counts for each of these pathway groupings compared to both the

411 GBDs. Only the cellulose group produced a significant effect of fiber dose on pathway

412 upregulation, as the 200CEL group demonstrated higher gene counts than the 100CEL group for

413 all the pathways except for carbohydrate degradation and absorption, which failed to reach

414 significance. Unlike inulin, neither of the FOS diets significantly affected the metabolic 
415 pathways compared to GBDs regardless of the dose. Fewer differences were observed in terms

416 of metabolic pathways in the colon samples (Supplementary Figure 2). PDs demonstrated

417 higher gene counts related to carbohydrate degradation and absorption, with the 200CEL, 200IN,

418 and the 200FOS groups reaching significantly higher levels compared to the GBDs. Galactose

419 metabolism was affected by 200CEL and 200FOS relative to GBDs. In the case of fructose and

420 mannose metabolism, starch and sucrose metabolism, fatty acid metabolism, and fatty acid

421 biosynthesis, only the 200CEL dietary treatment produced significantly higher gene counts

422 compared to all other groups. As in the cecum, 200CEL group increased gene counts compared

423 to the 100CEL group in most pathways except for carbohydrate degradation and absorption,

424 which failed to reach significance. The soluble fiber groups, regardless of dose, did not

425 significantly modulate metabolic pathway gene counts in the colon. 


\section{DISCUSSION}

439 PDs are an essential element of nutrition research and it is important to consider the effect that

440 background diet may have on the expressed phenotype. Since the AIN diets were designed 4

441 decades ago, we have learned more about how different nutrients alter metabolic profile of mice

442 and rats and this knowledge can be applied to improve these diets for future studies. he AIN

443 committee did allude to using a different source of carbohydrate in lieu of sucrose due to

444 potential influences of this ingredient on metabolic disorders, they did not make any specific

445 recommendations on adjusting the fiber levels in these $\operatorname{diets}(22)$. The current recommendation

446 for 5\% cellulose, an insoluble fiber, provides little fermentable dietary substrate accessible to the

447 intestinal microbiota. Current data clearly show that this lack of soluble fiber leads to dramatic

448 effects on gut and metabolic health in mice $(8,17)$. We therefore wished to further understand

449 how revised versions of the AIN diets with reduced sucrose and higher amounts of total fiber,

450 including soluble fiber, affected metabolic and gut health compared with GBDs.

452 In our chronic feeding study, fiber and sucrose alterations in the two OSDs used in this study did

453 not result in significant changes to overall adiposity index (combining all adipose depots relative

454 to carcass weight) relative to the GBD. Leptin was elevated in mice fed either the 93G or OSD

455 compared to those fed the GBD, suggesting that the reduced sucrose and fiber change had little

456 impact on these parameters. However, the addition of more fiber as cellulose suppressed this

457 effect and these mice had similar levels as those fed the GBD. While it is unknown how added

458 cellulose led to a reduced leptin level, a trend for reduced adipose depots in these animals

459 relative to other PDs may have partially accounted for this difference. Furthermore, there was

460 also a slight but significant increase in liver triglycerides in the OSD fed mice compared to those 
461 fed the GBD, while those fed the higher fiber OSD diet had similar levels as those fed GBD.

462 Overall, these changes suggested that total fiber content is more important to maintaining these

463 static biochemical parameters. However, our data suggested that the addition of inulin was key to

464 maintaining glucose tolerance and both OSD groups had similar glucose tolerance compared to

465 those fed the GBD. In addition, replacement of sucrose with glucose derived carbohydrates may

466 also have benefitted these mice fed OSD as sucrose may induce metabolic disease in rats and

467 mice $(23,24)$. While sucrose levels were reduced to $10 \%$ in the AIN-93G diet relative to $50 \%$ in

468 the AIN-76A diet, it was not completely removed due to pelleting and palatability concerns by

469 the AIN committee (25). However, even relatively lower levels of sucrose may elicit changes in

470 glucose tolerance over a chronic feeding period, which is due to the fructose component of this

471 carbohydrate (26).

472

473 Closer examination of how the type and amount of fiber in PDs influenced gut health (Study 2)

474 indicated that a replacement of cellulose with soluble fiber-based PD prevented the rapid cecum

475 and colon weight loss associated with traditional cellulose-based PDs. Both types of soluble fiber

476 at low and high doses were capable of maintaining cecum and colon weights relative to GBD-fed

477 animals. This phenomenon has also been observed by us previously in the context of a high-fat

478 diet, where an inulin-based high-fat PD helped to maintain cecum and colon weight changes

479 compared to cellulose-based high-fat PD. This effect was attributed to the microbiota as inulin's

480 ability to promote colon and cecum mass was completely absent in germ-free mice (17).

481 Alongside changes to organ morphology, soluble fiber based PDs caused rapid and dramatic

482 changes to the microbiota in contrast to GBDs, regardless of fiber content, including reduced

483 species richness (alpha diversity) in both cecum and colon tissues. This may be in part due to the 
484 differences in fiber diversity among these diets as GBDs contain diverse fiber contents, which

485 may support the growth of more diverse microbial species, leading to greater alpha diversity

486 values (27). Moreover, dietary intervention studies in humans in which only one fiber type is

487 added to the diet typically do not translate to increased alpha diversity metrics (28). Still, our

488 results indicate that cellulose alone was able to support greater species richness in a PD

489 compared to inulin and FOS. This could be partially because the fiber types present in GBDs are

490 predominantly insoluble types, like cellulose (4). Previous data suggest that cellulose is

491 important for age-related diversification of the intestinal microbiota (29) and thus our results

492 indicate that it may be better to continue including cellulose in future designs of PD. In fact, a

493 recent study suggested that adding inulin to a lower fat diet containing cellulose tended to

494 increase alpha diversity relative to cellulose alone, suggesting the importance of maintaining

495 cellulose to preserve species richness (30). Beta diversity analysis indicated that each fiber type

496 (GBDs, cellulose based, or soluble fiber based) supported distinctly different communities of

497 bacteria, regardless of fiber dose. These data also suggested that both GBDs supported growth of

498 similar microbial communities, which may not be surprising given they contain similar

499 ingredients and also insoluble and soluble fiber contents. This has been documented in other

500 studies and together, these findings indicate that single sources of purified fibers as part of a PD,

501 even at higher concentrations, may not support similar microbial species as the GBDs do. It is

502 likely the case that multiple purified fibers used in combination would optimally support the

503 microbiome and thus are necessary to achieve closer similarity to GBDs, regardless of the dose.

505 Although switching from GBD to PDs generally led to reduced species richness and supported

506 different microbial species compared to GBDs, some of the changes to dominant microbial taxa 
507 could be beneficial and ultimately yield better health outcomes in rodents, especially if soluble

508 fibers were to be added to a PD alongside cellulose in the future. The role of the Firmicutes to

509 Bacteriodota ratio in the onset of obesity itself remains controversial $(21,31)$. However,

510 increased relative abundance of Bacteriodota and other major phyla have demonstrated positive

511 health effects beyond obesity remediation. For example, a study by Rabot et al (31) indicated

512 that an increased abundance of Bacteriodota was associated with improved glucose tolerance in a

513 cohort of mice fed a high-fat diet. Moreover, it has been demonstrated that inclusion of prebiotic

514 fibers, like inulin and FOS, in the diet are linked with higher circulating levels of GLP-1, a

515 metabolic hormone with antidiabetic effects (32,33). Together, these observations could partially

516 explain why adding soluble fiber such as inulin to PDs is associated with improved metabolic

517 health compared to the insoluble fiber, cellulose based low-fiber, high-sucrose AIN diets as seen

518 from results of our first study.

520 There is a growing body of evidence suggesting that certain microbial genera positively

521 influence metabolic health. Akkermansia spp., which belong to the Verrucomicrobia phylum

522 (both of which were elevated due to the inclusion of soluble fiber in the diet), have demonstrated

523 the ability to maintain gut barrier integrity. As such, it is suspected that Akkermansia spp. may

524 exert anti-inflammatory properties, which in turn could contribute to overall metabolic health,

525 given that chronic inflammation is associated with insulin resistance and diabetes $(34,35)$. Other

526 studies have indicated that administration of FOS in rodent diets appears to increase

527 Akkermansia abundance in the gut (doses as low as $0.3 \mathrm{gm} / \mathrm{d}$, which translate roughly to $60 \mathrm{gm}$

528 FOS/kg diet), while diet-induced obesity appears to consistently reduce abundance of this genera

529 (36,37). Our study suggests that inulin and FOS may also increase the relative abundance of this 
530 metabolically active genus. Bifidobacteria, which are a subspecies of Actinobacteria have

531 historically been categorized as health promoting microbes (38). It is believed that these bacteria

532 play a role in stimulating host innate immunity, and may also enhance the ability of Bacteroides

533 to metabolize carbohydrates $(35,38)$. Bifidobacteria also appear to reduce gut permeability,

534 similarly to Akkermansia, and reductions in Bifidobacteria populations are found in diet-induced

535 obese rodents (39). The results from our study indicated that the addition of soluble fiber to a PD

536 can increase the abundance of Bifidobacteria relative to a GBD, and both cellulose and soluble

537 fibers may increase Bacteroides. Another microbial genus of interest is Roseburia, which is one

538 of the most abundant genera of the Firmicutes phylum. Despite general observations that overall

539 Firmicutes abundance is reduced in lean individuals relative to obese individuals, Roseburia spp.

540 tend to be more abundant in lean individuals (40). They also generally increase in relative

541 abundance in response to diets rich in FOS and inulin (41). However, in our study, all the

542 soluble-fiber based diets decreased Roseburia relative abundance compared to both GBDs and

543 cellulose based diets in the cecum and colon tissues. While these data are contrary to other

544 reported findings(41), it does support the notion that multiple fiber types may be necessary to

545 support the optimal growth of a consortium of beneficial bacteria. Roseburia spp. are well known

546 for their ability to produce high concentrations of butyrate, a potent short-chain fatty acid that is

547 known to promote colon health (42). Production of this metabolite, by Roseburia or other genera,

548 would certainly be indicative of the diet supporting a healthy gut. While short-chain fatty acids

549 were not measured in this study, previous collaborative studies suggested that replacement of

550 cellulose with around 5\% inulin in the context of a PD with low fat contents (similar to AIN and

551 OSD) elevated levels of fecal SCFA to a similar level as those fed a GBD (17). 
553 Further insights regarding the functional capacity of the microbiome in response to the dietary

554 treatments were explored using the PICRUSt program. Despite the limitation that this software

555 can only predict functional differences in the microbiota using marker-gene sequencing

556 techniques(43), several interesting trends with respect to the treatments were observed. Firstly, it

557 was evident from the metabolic pathways analysis (Figure 7 and Supplementary Figure 2) that

558 the dietary fibers, in particular the higher dose inulin group, had a greater impact on genes

559 related to metabolism in the cecums compared to the colons. The only group with significantly

560 higher metabolic pathway gene counts in both cecum and colon was the 200CEL group. This

561 could partially be explained by the fact that the cecum is the primary site of fermentation in

562 mice, so much of the inulin may have been rapidly fermented in the cecum, leaving little to no

563 substrate available for use by microbes in the latter portions of the gut (44). However, significant

564 differences in carbohydrate degradation and absorption were still found for inulin and FOS PDs

565 in the colon, suggesting that there was still enough remaining fructans from these sources for

566 microbes residing in the latter portions of the gut. Another partial explanation for the high-dose

567 cellulose group being the only treatment group significantly influencing colonic microbial

568 metabolism is the fact that cellulose is poorly fermented by non-ruminant mammals such as mice

569 and humans (45). Unlike the fast-fermenting soluble fibers, it was likely that still some

570 undigested cellulose passed into the colon, which could be utilized by the microbes residing

571 there. With respect to the functionality of the gut microbiome in response to the dietary

572 treatments, it is also worth noting that there appeared to be some dose-related effects, which

573 were not observed when looking at microbial abundance and diversity. Higher doses of cellulose

574 and inulin tended to increase gene counts in specific pathways to a greater extent than low-dose

575 counterparts in the cecum samples. In other words, greater substrate availability (i.e. higher 
576 dosage) translated to greater potential metabolic activity in these tissues. Taken together, all of

577 these findings indicate that cellulose likely plays some role in the metabolic functionality of the

578 gut microbiome, in part by maintaining microbial richness similar to GBDs, but soluble fiber

579 may help to support normal colon physiology and also promote the growth of health beneficial

580 microbial genera, which lead to improved gut morphology relative to cellulose alone. Overall,

581 higher concentrations of fiber support greater microbial activity compared to the AIN

582 recommended dose of $5 \%$ in a rodent diet.

584 In summary, it is clear that these changes to the formulation of traditional AIN PDs (increased

585 amount and addition of soluble fiber with replacement of sucrose with glucose derived

586 carbohydrate sources) provide certain improvements to metabolic health, which may have been

587 in part due to changes in the gut microbiota profile. However, to more closely mimic gut

588 microbiota in mice fed GBDs, the addition of multiple, diverse fiber sources will likely be

589 required (4). Our results suggest that a mixture of soluble and insoluble fiber types, present at

590 higher concentrations than the AIN formulations and without the addition of sucrose, may be

591 help to maintain microbial richness similar to a GBD while also supporting greater functionality

592 of the microbiome. However, it is difficult to say whether one type of soluble fiber is more

593 beneficial than the other and whether a change in fiber in a PD would allow for a similar

594 microbiome relative to all GBDs. Thus, a PD containing multiple sources of soluble fiber

595 including the ones not used in this study (for e.g. mannans, beta-glucan, hemicelluloses, pectin

596 etc.) may need to be developed. Future efforts should be directed towards determining the

597 optimal ratios of soluble and insoluble fibers in PDs, as well as exploring how these changes to

598 the gut microbiome may influence animal health in longer-term studies. It is also critical to focus 
not only on relative microbiome shifts and predicted functionality, but also to examine

600 concentrations of circulating microbial metabolites such as SCFAs to better understand the

601 potential benefits for metabolic health. Given the differences between PDs and GBDs, it should

602 be clear that these two diet types should not be compared against each other while determining

603 dietary effects on a given phenotype. This is particularly apparent when determining the

604 theoretical underpinnings of how dietary effects on gut health and the microbiome drive changes

605 in metabolic health outcomes, as suggested previously $(17,46,47)$. Unfortunately, GBDs are

606 frequently used as controls for many experimental studies testing effect of a PD (e.g. high-fat

607 diet studies), which leads to misinterpretation of results. While having a matched PD allows one

608 to compare how a given change in diet is altering the rodent phenotype, one particular concern

609 from the research community is that rodents consuming control PDs, although lower in fat, are

610 typically not as metabolically healthy as those fed a GBD. Thus, it is imperative that the research

611 community focus on improving the formulation to mitigate some of the adverse changes

612 associated with consumption of PDs. A metabolically healthy control PD would greatly help the

613 research community to decipher nutrient related phenotypic differences in a wide range of

614 scientific domains. While we mainly focused on sucrose levels and the type/concentration of the

615 fiber, future studies should also examine the type and level of fat in order to optimize the

616 formulation of a metabolically healthy PD, as recently discussed regarding the AIN series

617 formulas (8). While we understand that the current study is limited to certain metabolic

618 parameters and the use of prediction software to assess microbial functionality, this study

619 reinforces the notion that lab animal diets must be formulated and selected with utmost care, as

620 the gut microbiome is easily influenced by diet and shifts in the populations of microbes may

621 impact study outcomes. 


\section{ACKNOWLEDGEMENTS}

623 The authors would like to thank Dr. Kelly Swanson for his assistance with analyzing the fiber

624 content of the GBDs. We would also like to thank Alex La Reau at Diversigen for his assistance

625 with microbiome data analysis.

626

\section{AUTHOR CONTRIBUTIONS}

628 MP designed the studies and the diets, facilitated the completion of the studies with CROs, and

629 analyzed the data from Study 1. LG and SR wrote the manuscript and interpreted the data from

630 Study 2. All authors contributed to revisions and final submission of the manuscript. 


\section{REFERENCES}

1. Pellizzon MA, Ricci MR. Choice of Laboratory Rodent Diet May Confound Data Interpretation and Reproducibility. Current Developments in Nutrition Oxford University Press; 2020;4:nzaa031.

2. Wise A. Interaction of diet and toxicity — the future role of purified diet in toxicological research. Arch Toxicol 1982;50:287-99.

3. Pellizzon M, Radhakrishnan S, Ricci M. Mycotoxins and Endotoxin Content Vary Between Different Batches of Grain-Based Chow Diets. Baltimore, MD; 2019.

4. Pellizzon MA, Ricci MR. Effects of Rodent Diet Choice and Fiber Type on Data Interpretation of Gut Microbiome and Metabolic Disease Research. Current Protocols in Toxicology 2018;77:e55.

5. Reeves PG, Nielsen FH, Fahey GC. AIN-93 purified diets for laboratory rodents: final report of the American Institute of Nutrition ad hoc writing committee on the reformulation of the AIN-76A rodent diet. J Nutr 1993;123:1939-51.

6. Report of the American Institute of Nurtition ad hoc Committee on Standards for Nutritional Studies. Journal of Nutrition 107:1340-8.

7. Syed R, Shibata NM, Kharbanda KK, Su RJ, Olson K, Yokoyama A, Rutledge JC, Chmiel KJ, Kim K, Halsted CH, et al. Effects of Nonpurified and Choline Supplemented or Nonsupplemented Purified Diets on Hepatic Steatosis and Methionine Metabolism in C3H Mice. Metab Syndr Relat Disord 2016;14:202-9.

8. Klurfeld DM, Gregory JF III, Fiorotto ML. Should the AIN-93 Rodent Diet Formulas be Revised? The Journal of Nutrition 2021;151:1380-2.

9. González-Blázquez R, Alcalá M, Fernández-Alfonso MS, Villa-Valverde P, Viana M, GilOrtega M, Somoza B. Relevance of control diet choice in metabolic studies: impact in glucose homeostasis and vascular function. Sci Rep 2020;10:2902.

10. Wise A, Gilburt DJ. The variability of dietary fibre in laboratory animal diets and its relevance to the control of experimental conditions. Food Cosmet Toxicol 1980;18:643-8.

11. Sleder J, Chen YD, Cully MD, Reaven GM. Hyperinsulinemia in fructose-induced hypertriglyceridemia in the rat. Metabolism 1980;29:303-5.

12. Thresher JS, Podolin DA, Wei Y, Mazzeo RS, Pagliassotti MJ. Comparison of the effects of sucrose and fructose on insulin action and glucose tolerance. Am J Physiol Regul Integr Comp Physiol 2000;279:R1334-1340.

13. Pagliassotti MJ, Prach PA. Quantity of sucrose alters the tissue pattern and time course of insulin resistance in young rats. American Journal of Physiology-Regulatory, Integrative and Comparative Physiology American Physiological Society; 1995;269:R641-6. 
bioRxiv preprint doi: https://doi.org/10.1101/2022.02.28.482306; this version posted March 2, 2022. The copyright holder for this preprint

14. Flamm G, Glinsmann W, Kritchevsky D, Prosky L, Roberfroid M. Inulin and oligofructose as dietary fiber: a review of the evidence. Crit Rev Food Sci Nutr 2001;41:353-62.

15. Levrat MA, Rémésy C, Demigné C. High propionic acid fermentations and mineral accumulation in the cecum of rats adapted to different levels of inulin. J Nutr $1991 ; 121: 1730-7$.

16. Campbell JM, Fahey GC Jr, Wolf BW. Selected Indigestible Oligosaccharides Affect Large Bowel Mass, Cecal and Fecal Short-Chain Fatty Acids, $\mathrm{pH}$ and Microflora in Rats. The Journal of Nutrition 1997;127:130-6.

17. Chassaing B, Miles-Brown J, Pellizzon M, Ulman E, Ricci M, Zhang L, Patterson AD, Vijay-Kumar M, Gewirtz AT. Lack of soluble fiber drives diet-induced adiposity in mice. American Journal of Physiology-Gastrointestinal and Liver Physiology American Physiological Society; 2015;309:G528-41.

18. Zou J, Chassaing B, Singh V, Pellizzon M, Ricci M, Fythe MD, Kumar MV, Gewirtz AT. Fiber-Mediated Nourishment of Gut Microbiota Protects against Diet-Induced Obesity by Restoring IL-22-Mediated Colonic Health. Cell Host Microbe 2018;23:41-53.e4.

19. Brooks L, Viardot A, Tsakmaki A, Stolarczyk E, Howard JK, Cani PD, Everard A, Sleeth ML, Psichas A, Anastasovskaj J, et al. Fermentable carbohydrate stimulates FFAR2dependent colonic PYY cell expansion to increase satiety. Mol Metab 2017;6:48-60.

20. Roberfroid MB. Introducing inulin-type fructans. British Journal of Nutrition Cambridge University Press; 2005;93:S13-25.

21. Griffin LE, Witrick KA, Klotz C, Dorenkott MR, Goodrich KM, Fundaro G, McMillan RP, Hulver MW, Ponder MA, Neilson AP. Alterations to metabolically active bacteria in the mucosa of the small intestine predict anti-obesity and anti-diabetic activities of grape seed extract in mice. Food Funct 2017;8:3510-22.

22. Bieri JG. Second Report of the ad hoc Committee on Standards for Nutritional Studies. The Journal of Nutrition 1980;110:1726.

23. O’Brien P, Han G, Ganpathy P, Pitre S, Zhang Y, Ryan J, Sim PY, Harding SV, Gray R, Preedy VR, et al. Chronic Effects of a High Sucrose Diet on Murine Gastrointestinal Nutrient Sensor Gene and Protein Expression Levels and Lipid Metabolism. International Journal of Molecular Sciences Multidisciplinary Digital Publishing Institute; 2021;22:137.

24. Sumiyoshi M, Sakanaka M, Kimura Y. Chronic Intake of High-Fat and High-Sucrose Diets Differentially Affects Glucose Intolerance in Mice. The Journal of Nutrition 2006;136:5827.

25. Reeves PG. Components of the AIN-93 Diets as Improvements in the AIN-76A Diet. The Journal of Nutrition 1997;127:838S-841S. 
26. Baena M, Sangüesa G, Dávalos A, Latasa M-J, Sala-Vila A, Sánchez RM, Roglans N, Laguna JC, Alegret M. Fructose, but not glucose, impairs insulin signaling in the three major insulin-sensitive tissues. Sci Rep Nature Publishing Group; 2016;6:26149.

27. Singh RK, Chang H-W, Yan D, Lee KM, Ucmak D, Wong K, Abrouk M, Farahnik B, Nakamura M, Zhu TH, et al. Influence of diet on the gut microbiome and implications for human health. Journal of Translational Medicine 2017;15:73.

28. So D, Whelan K, Rossi M, Morrison M, Holtmann G, Kelly JT, Shanahan ER, Staudacher HM, Campbell KL. Dietary fiber intervention on gut microbiota composition in healthy adults: a systematic review and meta-analysis. The American Journal of Clinical Nutrition 2018;107:965-83.

29. Fischer F, Romero R, Hellhund A, Linne U, Bertrams W, Pinkenburg O, Eldin HS, Binder $\mathrm{K}$, Jacob R, Walker A, et al. Dietary cellulose induces anti-inflammatory immunity and transcriptional programs via maturation of the intestinal microbiota. Gut Microbes Taylor \& Francis; 2020;12:1829962.

30. Pontifex MG, Mushtaq A, Le Gall G, Rodriguez-Ramiro I, Blokker BA, Hoogteijling MEM, Ricci M, Pellizzon M, Vauzour D, Müller M. Differential Influence of Soluble Dietary Fibres on Intestinal and Hepatic Carbohydrate Response. Nutrients Multidisciplinary Digital Publishing Institute; 2021;13:4278.

31. Rabot S, Membrez M, Blancher F, Berger B, Moine D, Krause L, Bibiloni R, Bruneau A, Gérard P, Siddharth J, et al. High fat diet drives obesity regardless the composition of gut microbiota in mice. Sci Rep 2016;6:32484.

32. Hwang I, Park YJ, Kim Y-R, Kim YN, Ka S, Lee HY, Seong JK, Seok Y-J, Kim JB. Alteration of gut microbiota by vancomycin and bacitracin improves insulin resistance via glucagon-like peptide 1 in diet-induced obesity. The FASEB Journal 2015;29:2397-411.

33. Parnell JA, Reimer RA. Prebiotic fibres dose-dependently increase satiety hormones and alter Bacteroidetes and Firmicutes in lean and obese JCR:LA-cp rats. Br J Nutr 2012;107:601-13.

34. Zhou K. Strategies to promote abundance of Akkermansia muciniphila, an emerging probiotics in the gut, evidence from dietary intervention studies. J Funct Foods 2017;33:194-201.

35. Cani PD, Osto M, Geurts L, Everard A. Involvement of gut microbiota in the development of low-grade inflammation and type 2 diabetes associated with obesity. Gut Microbes Taylor \& Francis; 2012;3:279-88.

36. Reid DT, Eller LK, Nettleton JE, Reimer RA. Postnatal prebiotic fibre intake mitigates some detrimental metabolic outcomes of early overnutrition in rats. Eur J Nutr 2016;55:2399-409. 
37. Everard A, Lazarevic V, Gaïa N, Johansson M, Stla ahlman M, Backhed F, Delzenne NM, Schrenzel J, Francois P, Cani PD. Microbiome of prebiotic-treated mice reveals novel targets involved in host response during obesity. The ISME journal 2014;8:2116-30.

38. Lee J-H, O’Sullivan DJ. Genomic Insights into Bifidobacteria. Microbiology and Molecular Biology Reviews American Society for Microbiology; 2010;74:378-416.

39. Cani PD, Amar J, Iglesias MA, Poggi M, Knauf C, Bastelica D, Neyrinck AM, Fava F, Tuohy KM, Chabo C, et al. Metabolic endotoxemia initiates obesity and insulin resistance. Diabetes 2007;56:1761-72.

40. Ley RE, Turnbaugh PJ, Klein S, Gordon JI. Microbial ecology: Human gut microbes associated with obesity. Nature 2006;444:1022-3.

41. Vacca M, Celano G, Calabrese FM, Portincasa P, Gobbetti M, De Angelis M. The Controversial Role of Human Gut Lachnospiraceae. Microorganisms Multidisciplinary Digital Publishing Institute; 2020;8:573.

42. Tamanai-Shacoori Z, Smida I, Bousarghin L, Loreal O, Meuric V, Fong SB, BonnaureMallet M, Jolivet-Gougeon A. Roseburia spp.: a marker of health? Future Microbiology Future Medicine; 2017;12:157-70.

43. Douglas GM, Beiko RG, Langille MGI. Predicting the Functional Potential of the Microbiome from Marker Genes Using PICRUSt. Methods Mol Biol 2018;1849:169-77.

44. Brown K, Abbott DW, Uwiera RRE, Inglis GD. Removal of the cecum affects intestinal fermentation, enteric bacterial community structure, and acute colitis in mice. Gut Microbes 2018;9:218-35.

45. Kim Y, Hwang SW, Kim S, Lee Y-S, Kim T-Y, Lee S-H, Kim SJ, Yoo HJ, Kim EN, Kweon M-N. Dietary cellulose prevents gut inflammation by modulating lipid metabolism and gut microbiota. Gut Microbes Taylor \& Francis; 2020;11:944-61.

46. Dalby MJ, Ross AW, Walker AW, Morgan PJ. Dietary Uncoupling of Gut Microbiota and Energy Harvesting from Obesity and Glucose Tolerance in Mice. Cell Reports Elsevier; 2017;21:1521-33.

47. Daniel N, Rossi Perazza L, Varin TV, Trottier J, Marcotte B, St-Pierre P, Barbier O, Chassaing B, Marette A. Dietary fat and low fiber in purified diets differently impact the gut-liver axis to promote obesity-linked metabolic impairments. American Journal of Physiology-Gastrointestinal and Liver Physiology American Physiological Society; 2021;320:G1014-33. 


\section{TABLES}

Table 1: Composition of the PDs used for Study 1

Table 2: Composition of PDs used in Study 2

Table 3: Fat pad and carcass weights of the mice at the end of the study 1 after 88 days on the experimental diets.

Table 4: Biochemical measures of serum and liver of the mice at the end of the study 1 after 88 days on the experimental diets.

Table 5: Body weights, colon lengths and cecum/colon weights of the mice at the end of the study 2 after 14 days on the experimental diets (Study 2)

Supplementary Table 1: P-values for individual microbial taxa differences (pairwise comparisons, Tukey HSD) at the phylum level in the cecum samples.

Supplementary Table 2: P-values for individual microbial taxa differences (pairwise comparisons, Tukey HSD) at the phylum level in the colon samples.

Supplementary Table 3: P-values for individual microbial taxa differences (pairwise comparisons, Tukey HSD) at the genus level in the cecum samples.

Supplementary Table 4: P-values for individual microbial taxa differences (pairwise comparisons, Tukey HSD) at the genus level in the colon samples.

\section{FIGURE LEGENDS}

Figure 1. (Study 1) A. Mouse body weight measurements over an 88 day timespan expressed as means 土 SEM and B. Final body weight measurements expressed as means 土 SEM. Groups with different letters represent significantly different results by 1-way ANOVA with Tukey's HSD post hoc analysis $(\mathrm{p}<0.05)$. Mouse weights were recorded weekly over the 88 day 
metabolic phenotype study for each of the 5 dietary treatment groups $(n=15 /$ treatment $)$ OSD, OSD + F, 76A, 93G, and chow.

Figure 2: (Study 1). A. Blood glucose measurements over time following a 6-hr fast and an oral glucose load, with data points representing means 土 SEM for each treatment group. B. Blood glucose area under the curve on day 83. Groups with different letters represent significantly different results by 1 -way ANOVA with Tukey's HSD post hoc analysis $(\mathrm{p}<0.05)$ for each of the 5 dietary treatment groups $(n=15 /$ treatment $)$ OSD, OSD $+F, 76 \mathrm{~A}, 93 \mathrm{G}$, and chow.

Figure 3. (Study 2). Images of representative cecums and colons for each dietary treatment group after 14 days on either a GBD (5001 or 5002), or high-fiber PDs (100CEL, 100IN, 100FOS, 200CEL, 200IN, 200FOS).

Figure 4. (Study 2). Alpha diversity measurements for Shannon index (A/D), Chao1 index (B/E) and Observed ASVs (C/F) by dietary treatment group for colon (A-C) and cecum (D-F) samples. Data are expressed as means 土 SEM for each treatment group. Groups with different letters represent significantly different results by one-way ANOVA with Tukey's post hoc analysis $(\mathrm{p}<$ 0.05) after 14 days on either a GBD (5001 or 5002), or high-fiber PDs (100CEL, 100IN, 100FOS, 200CEL, 200IN, 200FOS) with $n=7-8$ /group.

Figure 5. (Study 2). Bray-Curtis Dissimilarity plots for colon (A) and cecum (B) samples after 14 days on either a GBD (5001 or 5002), or high-fiber PDs (100CEL, 100IN, 100FOS, 200CEL, 200IN, 200FOS) with $n=7-8$ /group. 
Figure 6. (Study 2). Composition of fiber in the diet modulates the composition of the gut microbiota. Relative abundance of dominant phyla by dietary treatment for cecums (A) and colons (B) and at the ASV level for cecums (C) and colon (D) after 14 days on either a GBD (5001 or 5002), or high-fiber PDs (100CEL, 100IN, 100FOS, 200CEL, 200IN, 200FOS) with n $=8 /$ group .

Figure 7. (Study 2). Predicted carbohydrate and lipid metabolism of bacterial communities in the cecum using the PICRUSt program after 14 days on either a GBD (5001 or 5002), or high-fiber PDs (100CEL, 100IN, 100FOS, 200CEL, 200IN, 200FOS) with $n=8$ /group. (A) Carbohydrate digestion and absorption; (B) fructose and mannose metabolism; (C) galactose metabolism; (D) starch and sucrose metabolism; (E) fatty acid metabolism; (F) fatty acid biosynthesis. Data are expressed as means 土 SEM for each treatment group. Groups with different letters represent significantly different results by 1 -way ANOVA with Tukey's HSD post hoc analysis $(\mathrm{p}<0.05)$.

Supplementary Figure 1: (Study 2). Composition of fiber in the diet modulates the composition of the gut microbiota. Changes in Firmicutes/Bacteroidetes ratio in different groups dietary treatment for cecums (A) and colons (B) after 14 days on either a GBD (5001 or 5002), or highfiber PDs (100CEL, 100IN, 100FOS, 200CEL, 200IN, 200FOS) with $\mathrm{n}=8$ /group.

Supplementary Figure 2. (Study) Predicted carbohydrate and lipid metabolism of bacterial communities in the colon using the PICRUSt program after 14 days on either a GBD (5001 or 5002), or high-fiber PDs (100CEL, 100IN, 100FOS, 200CEL, 200IN, 200FOS) with n = 
bioRxiv preprint doi: https://doi.org/10.1101/2022.02.28.482306; this version posted March 2, 2022. The copyright holder for this preprint (which was not certified by peer review) is the author/funder, who has granted bioRxiv a license to display the preprint in perpetuity. It is made available under aCC-BY-ND 4.0 International license.

6/group. (A) Carbohydrate digestion and absorption; (B) fructose and mannose metabolism; (C) galactose metabolism; (D) starch and sucrose metabolism; (E) fatty acid metabolism; (F) fatty acid biosynthesis. Data are expressed as means 土 SEM for each treatment group. Groups with different letters represent significantly different results by 1-way ANOVA with Tukey's HSD post hoc analysis $(\mathrm{p}<0.05)$. 
bioRxiv preprint doi: $\mathrm{https}$ //doi.org/10.1101/2022.02.28.482306: this version posted March 2, 2022. The copyright holder for this preprint (which was not certified by peer review) is the author/funder, who has granted bioRxiv a license to display the preprint in perpetuity. It is made available under aCC-BY-ND 4.0 International license.

\begin{tabular}{|c|c|c|c|c|c|c|c|c|}
\hline & & & \multicolumn{6}{|c|}{ Table 1: Composition of the PDs used for Study 1} \\
\hline \multirow[t]{3}{*}{ Product \# } & \multirow{2}{*}{\multicolumn{2}{|c|}{$\begin{array}{r}\text { D10001 } \\
\text { AIN-76A }\end{array}$}} & \multicolumn{2}{|c|}{ D10012G } & \multicolumn{2}{|c|}{ D11112201 } & \multicolumn{2}{|c|}{ D11112202 } \\
\hline & & & AIN- & & OS & & OSI & \\
\hline & gm\% & kcal\% & gm\% & kcal\% & $\mathbf{g m} \%$ & kcal\% & $\mathbf{g m} \%$ & kcal\% \\
\hline Protein & 20 & 21 & 20 & 20 & 19 & 20 & 17 & 20 \\
\hline Carbohydrate & 66 & 68 & 64 & 64 & 63 & 65 & 42 & 65 \\
\hline Fat & 5 & 12 & 7 & 16 & 7 & 15 & 6 & 15 \\
\hline Total & & 100 & & 100 & & 100 & & 100 \\
\hline $\mathrm{kcal} / \mathrm{gm}$ & 3.90 & & 4.00 & & 3.81 & & 3.34 & \\
\hline Ingredient & gm & kcal & gm & kcal & gm & kcal & gm & kcal \\
\hline & & & & & & & & \\
\hline Casein & 200 & 800 & 200 & 800 & 200 & 800 & 200 & 800 \\
\hline DL-Methionine & 3 & 12 & 0 & 0 & 0 & 0 & 0 & 0 \\
\hline L-Cystine & 0 & 0 & 3 & 12 & 3 & 12 & 3 & 12 \\
\hline & & & & & & & & \\
\hline Corn Starch & 150 & 600 & 397.486 & 1590 & 381 & 1524 & 381 & 1524 \\
\hline Maltodextrin & 0 & 0 & 132 & 528 & 110 & 440 & 110 & 440 \\
\hline Sucrose & 500 & 2000 & 100 & 400 & 0 & 0 & 0 & 0 \\
\hline Dextrose, Monohydrate & 0 & 0 & 0 & 0 & 150 & 600 & 150 & 600 \\
\hline Cellulose (Insoluble Fiber) & 50 & 0 & 50 & 0 & 75 & 0 & 225 & 0 \\
\hline Inulin (Soluble Fiber) & 0 & 0 & 0 & 0 & 25 & 38 & 25 & 38 \\
\hline Corn Oil & 50 & 450 & 0 & 0 & 0 & 0 & 0 & 0 \\
\hline Soybean Oil & 0 & 0 & 70 & 630 & 70 & 630 & 70 & 630 \\
\hline t-BHQ & 0 & 0 & 0.014 & 0 & 0 & 0 & 0 & 0 \\
\hline & & & & & & & & \\
\hline Mineral Mix S10001 & 35 & 0 & 0 & 0 & 0 & 0 & 0 & 0 \\
\hline Mineral Mix S10022G & 0 & 0 & 35 & 0 & 0 & 0 & 0 & 0 \\
\hline Mineral Mix S10026 & 0 & 0 & 0 & 0 & 10 & 0 & 10 & 0 \\
\hline Dicalcium Phosphate & 0 & 0 & 0 & 0 & 13 & 0 & 13 & 0 \\
\hline Calcium Carbonate & 0 & 0 & 0 & 0 & 5.5 & 0 & 5.5 & 0 \\
\hline Potassium Citrate, $1 \mathrm{H} 2 \mathrm{O}$ & 0 & 0 & 0 & 0 & 16.5 & 0 & 16.5 & 0 \\
\hline Vitamin Mix V10001, AIN-76A & 10 & 40 & 0 & 0 & 10 & 40 & 10 & 40 \\
\hline Vitamin Mix V10037, AIN-93G & 0 & 0 & 10 & 40 & 0 & 0 & 0 & 0 \\
\hline Choline Bitartrate & 2 & 0 & 2.5 & 0 & 2 & 0 & 2 & 0 \\
\hline Yellow Dye \#5, FD\&C & 0 & 0 & 0 & 0 & 0.025 & 0 & 0 & 0 \\
\hline Red Dye $\# 40$, FD\&C & 0 & 0 & 0 & 0 & 0 & 0 & 0.05 & 0 \\
\hline Blue Dye \#1, FD\&C & 0 & 0 & 0 & 0 & 0.025 & 0 & 0 & 0 \\
\hline Total & 1000 & 3902 & 1000 & 4000 & 1071.05 & 4084 & 1221.05 & 4084 \\
\hline
\end{tabular}




\begin{tabular}{|c|c|c|c|c|c|c|c|c|c|c|c|c|}
\hline \multicolumn{13}{|c|}{ Table 2: Composition of PDs used in Study 2} \\
\hline \multirow[t]{4}{*}{ Product \# } & \multicolumn{2}{|c|}{ D11112222 } & \multicolumn{2}{|c|}{ D11112223 } & \multicolumn{2}{|c|}{ D11112224 } & \multicolumn{2}{|c|}{ D11112225 } & \multicolumn{2}{|c|}{ D11112226 } & \multicolumn{2}{|c|}{ D11112227 } \\
\hline & \multicolumn{2}{|c|}{ OSD+200 g Cellulose } & \multicolumn{2}{|c|}{$O S D+200 \mathrm{~g}$ Inulin } & \multicolumn{2}{|c|}{$O S D+200 \mathrm{~g} F O S$} & \multicolumn{2}{|c|}{ OSD $+100 \mathrm{~g}$ Cellulose } & \multicolumn{2}{|c|}{ OSD $+100 \mathrm{~g}$ Inulin } & \multicolumn{2}{|c|}{$O S D+100 g$ FOS } \\
\hline & \multicolumn{2}{|c|}{$200 C E L$} & \multicolumn{2}{|c|}{$200 I N$} & \multicolumn{2}{|c|}{$200 \mathrm{FOS}$} & \multicolumn{2}{|c|}{$100 C E L$} & \multicolumn{2}{|c|}{$100 I N$} & \multicolumn{2}{|c|}{$100 \mathrm{FOS}$} \\
\hline & $\mathrm{gm} \%$ & kcal\% & gm\% & kcal\% & gm\% & kcal\% & & kcal\% & $\mathrm{gm} \%$ & kcal\% & gm\% & kcal\% \\
\hline Protein & 17.2 & 20 & 18.4 & 20 & 18.4 & 20 & 18.8 & 20 & 19.5 & 20 & 19.5 & 20 \\
\hline Carbohydrate & 55.9 & 65 & 71.1 & 65 & 53.0 & 57 & 61.1 & 65 & 69.3 & 65 & 59.7 & 61 \\
\hline Fat & 5.9 & 15 & 6.3 & 15 & 6.3 & 15 & 6.5 & 15 & 6.7 & 15 & 6.7 & 15 \\
\hline Total & & 100 & & 100 & & 100 & & 100 & & 100 & & 100 \\
\hline \multirow[t]{2}{*}{$\mathrm{kcal} / \mathrm{gm}$} & 3.46 & & 3.69 & & 3.69 & & 3.78 & & 3.92 & & 3.92 & \\
\hline & & & & & & & & & & & & \\
\hline Ingredient & gm & kcal & $\mathbf{g m}$ & kcal & gm & kcal & gm & kcal & gm & kcal & $\mathbf{g m}$ & kcal \\
\hline Casein & 200 & 800 & 200 & 800 & 200 & 800 & 200 & 800 & 200 & 800 & 200 & 800 \\
\hline \multirow[t]{2}{*}{ L-Cystine } & 3 & 12 & 3 & 12 & 3 & 12 & 3 & 12 & 3 & 12 & 3 & 12 \\
\hline & & & & & & & & & & & & \\
\hline Corn Starch & 390.5 & 1562 & 315.5 & 1262 & 315.5 & 1262 & 390.5 & 1562 & 353 & 1412 & 353 & 1412 \\
\hline Maltodextrin 10 & 110 & 440 & 110 & 440 & 110 & 440 & 110 & 440 & 110 & 440 & 110 & 440 \\
\hline Dextrose & 150 & 600 & 150 & 600 & 150 & 600 & 150 & 600 & 150 & 600 & 150 & 600 \\
\hline & & & & & & & & & & & & \\
\hline Cellulose & 200 & 0 & 0 & 0 & 0 & 0 & 100 & 0 & 0 & 0 & 0 & 0 \\
\hline Inulin & 0 & 0 & 200 & 300 & 0 & 0 & 0 & 0 & 100 & 150 & 0 & 0 \\
\hline Fructooligosaccharide & 0 & 0 & 0 & 0 & 200 & 300 & 0 & 0 & 0 & 0 & 100 & 150 \\
\hline & & & & & & & & & & & & \\
\hline Soybean Oil & 70 & 630 & 70 & 630 & 70 & 630 & 70 & 630 & 70 & 630 & 70 & 630 \\
\hline & & & & & & & & & & & & \\
\hline Mineral Mix S10026 & 10 & 0 & 10 & 0 & 10 & 0 & 10 & 0 & 10 & 0 & 10 & 0 \\
\hline Dicalcium Phosphate & 13 & 0 & 13 & 0 & 13 & 0 & 13 & 0 & 13 & 0 & 13 & 0 \\
\hline \begin{tabular}{|l} 
Calcium Carbonate \\
\end{tabular} & 5.5 & 0 & 5.5 & 0 & 5.5 & 0 & 5.5 & 0 & 5.5 & 0 & 5.5 & 0 \\
\hline Potassium Citrate, $1 \mathrm{H} 2 \mathrm{O}$ & 16.5 & 0 & 16.5 & 0 & 16.5 & 0 & 16.5 & 0 & 16.5 & 0 & 16.5 & 0 \\
\hline & & & & & & & & & & & & \\
\hline Vitamin Mix V10001 & 10 & 40 & 10 & 40 & 10 & 40 & 10 & 40 & 10 & 40 & 10 & 40 \\
\hline Choline Bitartrate & 2 & 0 & 2 & 0 & 2 & 0 & 2 & 0 & 2 & 0 & 2 & 0 \\
\hline & & & & & & & & & & & & \\
\hline Yellow Dye $\# 5$, FD\&C & 0.05 & 0 & 0 & 0 & 0 & 0 & 0.025 & 0 & 0 & 0 & 0.01 & 0 \\
\hline Red Dye \#40, FD\&C & 0 & 0 & 0.05 & 0 & 0 & 0 & 0.025 & 0 & 0.025 & 0 & 0.04 & 0 \\
\hline Blue Dye $\# 1$, FD\&C & 0 & 0 & 0 & 0 & 0.05 & 0 & 0 & 0 & 0.025 & 0 & 0 & 0 \\
\hline & & & & & & & & & & & & \\
\hline Total & 11180.55 & 4084 & 1105.55 & 4084 & 1105.55 & 4084 & \begin{tabular}{|l|}
1080.55 \\
\end{tabular} & 4084 & \begin{tabular}{|l|}
1043.05 \\
\end{tabular} & 4084 & 1043.05 & 4084 \\
\hline & & & & & & & & & & & & \\
\hline Total Fiber (\%) & 16.9 & & 18.1 & & 18.1 & & 9.3 & & 9.6 & & 9.6 & \\
\hline Total Insoluble (\%) & 16.9 & & $\mathbf{0}$ & & $\mathbf{0}$ & & 9.3 & & $\mathbf{0}$ & & $\mathbf{0}$ & \\
\hline Total Soluble (\%) & $\mathbf{0}$ & & 18.1 & & 18.1 & & 0 & & 9.6 & & 9.6 & \\
\hline
\end{tabular}


Table 3. Fat Pad and Carcass Weights in Study 1

\begin{tabular}{lcccccc}
\hline & OSD & OSD + F & 93G & 76A & Chow & p-value \\
\cline { 2 - 6 } Messenteric Fat Pad (g) & $0.55(0.03)$ & $0.48(0.04)$ & $0.46(0.07)$ & $0.48(0.05)$ & $0.45(0.04)$ & 0.5388 \\
Gonadal Fat pad (g) & $1.81(0.10)^{\mathrm{a}}$ & $1.41(0.07)^{\mathrm{ab}}$ & $1.67(0.16)^{\mathrm{ab}}$ & $1.39(0.14)^{\mathrm{ab}}$ & $1.30(0.11)^{\mathrm{b}}$ & 0.0158 \\
Retroperitoneal Fat Pad (g) & $0.54(0.04)$ & $0.50(0.05)$ & $0.45(0.05)$ & $0.50(0.05)$ & $0.43(0.04)$ & 0.3193 \\
Inguinal Fat Pad (g) & $1.61(0.12)^{\mathrm{a}}$ & $1.39(0.1)^{\mathrm{ab}}$ & $1.0(0.13)^{\mathrm{b}}$ & $1.14(0.13)^{\mathrm{b}}$ & $1.27(0.11)^{\mathrm{ab}}$ & 0.0086 \\
Total Fat (g) & $4.52(0.25)^{\mathrm{a}}$ & $3.78(0.21)^{\mathrm{ab}}$ & $3.58(0.3)^{\mathrm{ab}}$ & $3.52(0.31)^{\mathrm{ab}}$ & $3.45(0.28)^{\mathrm{b}}$ & 0.0425 \\
Carcass Weight (g) & $32.2(1.1)^{\mathrm{a}}$ & $29.6(0.8)^{\mathrm{ab}}$ & $31.3(1.1)^{\mathrm{ab}}$ & $27.8(1.1)^{\mathrm{b}}$ & $29.7(0.9)^{\mathrm{ab}}$ & 0.0231 \\
Adiposity Index (g fat/g carcass) & $13.9(0.5)^{\mathrm{a}}$ & $12.7(0.6)^{\mathrm{ab}}$ & $11.0(0.7)^{\mathrm{b}}$ & $12.3(0.8)^{\mathrm{ab}}$ & $11.5(0.7)^{\mathrm{ab}}$ & 0.0486 \\
\hline
\end{tabular}

Data are expressed as means(SEM). Different letters across rows represent significantly different values

Table 4. Terminal Serum and Liver Data in Study 1

\begin{tabular}{lcccccc}
\hline & OSD & OSD + F & 93G & 76A & Chow & p-value \\
\cline { 2 - 6 } Serum Insulin (ng/mL) & $4.6(0.6)$ & $3.4(0.4)$ & $5.2(0.6)$ & $3.3(0.6)$ & $3.5(0.5)$ & 0.0378 \\
Serum Glucose (mg/dL) & $267.8(16.4)$ & $244.1(12.9)$ & $254.7(8.9)$ & $251.0(15.9)$ & $236.0(11.3)$ & 0.5248 \\
Serum Triglyceride (mg/dL) & $96.5(4.4)^{\mathrm{ab}}$ & $92.2(2.7)^{\mathrm{ab}}$ & $92.7(3.2)^{\mathrm{ab}}$ & $80.9(0.05)^{\mathrm{b}}$ & $102.7(3.2)^{\mathrm{a}}$ & 0.0031 \\
Serum Cholesterol (mg/dL) & $166.7(10.2)^{\mathrm{a}}$ & $171.9(5.3)^{\mathrm{a}}$ & $223.5(6.2)^{\mathrm{c}}$ & $170.6(9.0)^{\mathrm{a}}$ & $129.7(2.6)^{\mathrm{b}}$ & $<0.0001$ \\
Serum Leptin (ng/mL) & $34.3(1.2)^{\mathrm{a}}$ & $23.5(2.6)^{\mathrm{b}}$ & $35.6(2.4)^{\mathrm{a}}$ & $26.2(3.0)^{\mathrm{ab}}$ & $22.2(2.6)^{\mathrm{b}}$ & 0.0002 \\
Liver Weight (g) & $1.55(0.10)$ & $1.39(0.08)$ & $1.36(0.08)$ & $1.36(0.06)$ & $1.57(0.04)$ & 0.1096 \\
Liver Triglyceride (mg/g) & $10.1(1.0)^{\mathrm{ab}}$ & $8.2(0.9)^{\mathrm{abc}}$ & $10.5(1.3)^{\mathrm{a}}$ & $6.5(1.0)^{\mathrm{bc}}$ & $4.5(0.3)^{\mathrm{c}}$ & $<0.0001$ \\
\hline
\end{tabular}

Data are expressed as means(SEM). Different letters across rows represent significantly different values

Table 5. Body and Organ Weights at Baseline and After 2 Weeks on a GB or Purified OSD in Study 2

Day 1 Body Weight (g)

Day 14 Body Weight (g)

$\%$ Change Bodyweight

Colon Length $(\mathrm{cm})$

$\%$ Change Colon Length

Cecum+Colon Weight $(\mathrm{g} / 100 \mathrm{~g} \mathrm{BW})$

$\%$ Change in Cecum + Colon Weight

Cecum + Colon Content Weight $(\mathrm{g} / 100 \mathrm{~g} \mathrm{BW})$

$\%$ Change in Cecum + Colon Content Weight

Cecum Weight (g/100 g BW)

$\%$ Change in Cecum Content Weight

Cecum Content Weight (g/100 g BW)

$\%$ Change in Cecum Content Weight

Colon Weight (g/100 g BW)

$\%$ Change in Colon Weight

Colon Contents (g/100 g BW)

$\%$ Change in Colon Content Weight

Data are expressed as means(SEM). Asteri

\begin{tabular}{|c|c|c|c|c|c|c|c|c|c|}
\hline Baseline & 5001 & 5002 & 100CEL & $100 I N$ & 100FOS & 200CEL & $200 I N$ & $200 \mathrm{FOS}$ & p-value \\
\hline \multirow[t]{3}{*}{$17(1)$} & $16.5(0.6)$ & $16.2(1.3)$ & $15.3(0.2)$ & $15.3(0.2)$ & $15.5(0.4)$ & $15.2(0.3)$ & $15.8(0.6)$ & $16.7(0.7)$ & 0.4052 \\
\hline & $23.2(0.4)^{\mathrm{a}}$ & $23.5(0.6)^{\mathrm{a}}$ & $22.7(0.3)^{\mathrm{ab}}$ & $21.5(0.6)^{\mathrm{ab}}$ & $22.3(0.8)^{\mathrm{ab}}$ & $20.8(0.6)^{\mathrm{ab}}$ & $21.5(0.6)^{\mathrm{ab}}$ & $20.3(0.9)^{b}$ & $<0.0001$ \\
\hline & $41(5)$ & $52(17)$ & $48(4)$ & $39(5)$ & $54(10)$ & $38(4)$ & $37(5)$ & $25(6)$ & 0.1671 \\
\hline \multirow[t]{2}{*}{$5.6(02)$} & $7.9(0.2)^{* a b}$ & $8.7(0.3)^{* a}$ & $6.8(0.4)^{b}$ & $7.8(0.3)^{* a b}$ & $7.0(0.4)^{* b}$ & $7.6(0.4)^{\mathrm{ab}}$ & $8.0(0.2)^{\mathrm{ab}}$ & $8.3(0.3)^{\mathrm{ab}}$ & $<0.0001$ \\
\hline & 41 & 56 & 22 & 38 & 25 & 35 & 42 & 47 & \\
\hline \multirow[t]{2}{*}{$2.3(0.3)$} & $1.5(0.1)^{* \mathrm{a}}$ & $1.5(0.1)^{* \mathrm{a}}$ & $1.0(0.03)^{* \mathrm{~b}}$ & $1.5(0.1)^{* \mathrm{a}}$ & $1.4(0.1)^{* a b}$ & $1.2(0.1)^{* a b}$ & $2.3(0.1)^{\mathrm{c}}$ & $2.1(0.2)^{\mathrm{c}}$ & $<0.0001$ \\
\hline & -36 & -36 & -59 & -36 & -41 & -50 & 1 & -9 & \\
\hline \multirow[t]{2}{*}{$2.8(0.4)$} & $2.1(0.1)^{\mathrm{a}}$ & $2.5(0.2)^{\mathrm{ac}}$ & $1.2(0.1)^{* b}$ & $2.8(0.1)^{\mathrm{ad}}$ & $2.0(0.1)^{* a}$ & $1.0(0.04)^{* b}$ & $3.7(0.2)^{*}$ & $3.2(0.3)^{\mathrm{cd}}$ & $<0.0001$ \\
\hline & -25 & -9 & -57 & 1 & -30 & -64 & 31 & 14 & \\
\hline \multirow[t]{2}{*}{$1.6(0.3)$} & $0.67(0.1)^{* a}$ & $0.69(0.04)^{* a}$ & $0.37(0.02)^{* a}$ & $0.73(0.1)^{* a}$ & $0.69(0.1)^{*_{a}}$ & $0.44(0.02)^{* a}$ & $1.4(0.1)^{b}$ & $1.2(0.2)^{\mathrm{b}}$ & $<0.0001$ \\
\hline & -57 & -58 & -77 & -54 & -57 & -73 & -11 & -23 & \\
\hline \multirow[t]{2}{*}{$2.2(0.4)$} & $1.6(0.1)^{\mathrm{ab}}$ & $1.9(0.1)^{\mathrm{ac}}$ & $0.8(0.1)^{* \mathrm{~b}}$ & $2.3(0.2)^{\mathrm{ac}}$ & $1.7(0.1)^{\mathrm{ac}}$ & $0.7(0.1)^{\mathrm{ac}}$ & $3.1(0.2)^{*_{\mathrm{c}}}$ & $2.5(0.4)^{\mathrm{c}}$ & $<0.0001$ \\
\hline & -30 & -13 & -63 & 5 & -69 & -70 & 39 & 14 & \\
\hline \multirow[t]{2}{*}{$0.35(0.05)$} & $0.77(0.02)^{* a c}$ & $0.8(0.1)^{* a c}$ & $0.57(0.04)^{* b}$ & $0.73(0.04)^{* a b c}$ & $0.67(0.1)^{* a b}$ & $0.71(0.1)^{* a b c}$ & $0.91(0.1)^{* \mathrm{c}}$ & $0.86(0.1)^{* a c}$ & $<0.0001$ \\
\hline & 122 & 128 & 65 & 110 & 91 & 104 & 161 & 148 & \\
\hline \multirow[t]{2}{*}{$0.57(0.1)$} & $0.52(0.03)^{\mathrm{abc}}$ & $0.61(0.1)^{\mathrm{ac}}$ & $0.39(0.04)^{\mathrm{abc}}$ & $0.49(0.1)^{\mathrm{abc}}$ & $0.28(0.1)^{* b}$ & $0.35(0.1)^{\mathrm{ab}}$ & $0.57(0.1)^{* b}$ & $0.65(0.13)^{\mathrm{c}}$ & 0.002 \\
\hline & -8 & 8 & -31 & -14 & -52 & -39 & 0 & 15 & \\
\hline
\end{tabular}
$-39$ 15 
Figure 1. (Study 1) A. Mouse body weight measurements over an 88 day timespan expressed as means 土 SEM and B. Final body weight measurements expressed as means 土 SEM. Groups with different letters represent significantly different results by 1-way ANOVA with Tukey's HSD post hoc analysis $(\mathrm{p}<0.05)$. Mouse weights were recorded weekly over the 88 day metabolic phenotype study for each of the 5 dietary treatment groups $(\mathrm{n}=15 /$ treatment): OSD, OSD + F, 76A, 93G, and chov

A

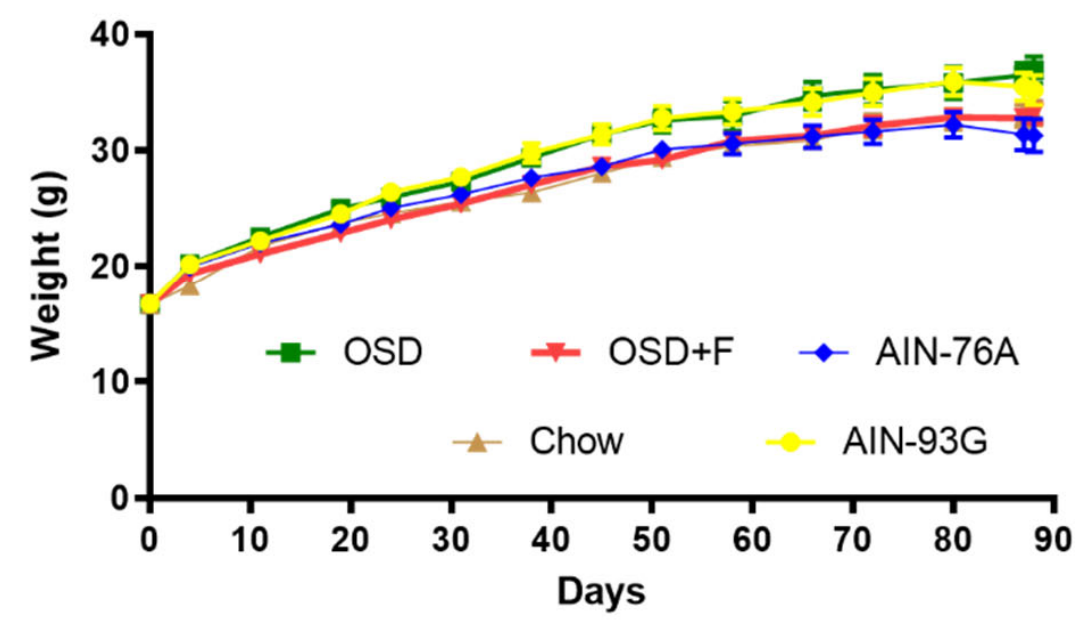

B Day 88 Body Weight

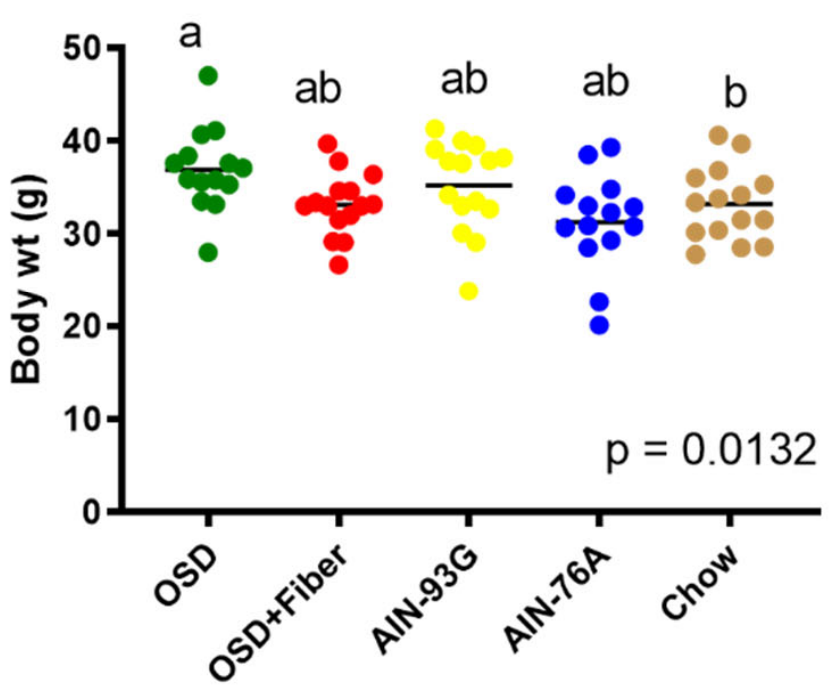

- OSD

- OSD+F

- Chow

AIN-93G

- AIN-76A 
Figure 2: (Study 1). A. Blood glucose measurements over time following a 6-hr fast and an oral glucose load, with data points representing means 土 SEM for each treatment group. B. Blood glucose area under the curve on day 83.

Groups with different letters represent significantly different results by 1-way ANOVA with Tukey's HSD post hoc analysis $(\mathrm{p}<0.05)$ for each of the 5 dietary treatment groups $(n=15 /$ treatment): OSD, OSD $+F, 76 \mathrm{~A}, 93 \mathrm{G}$, and chow.
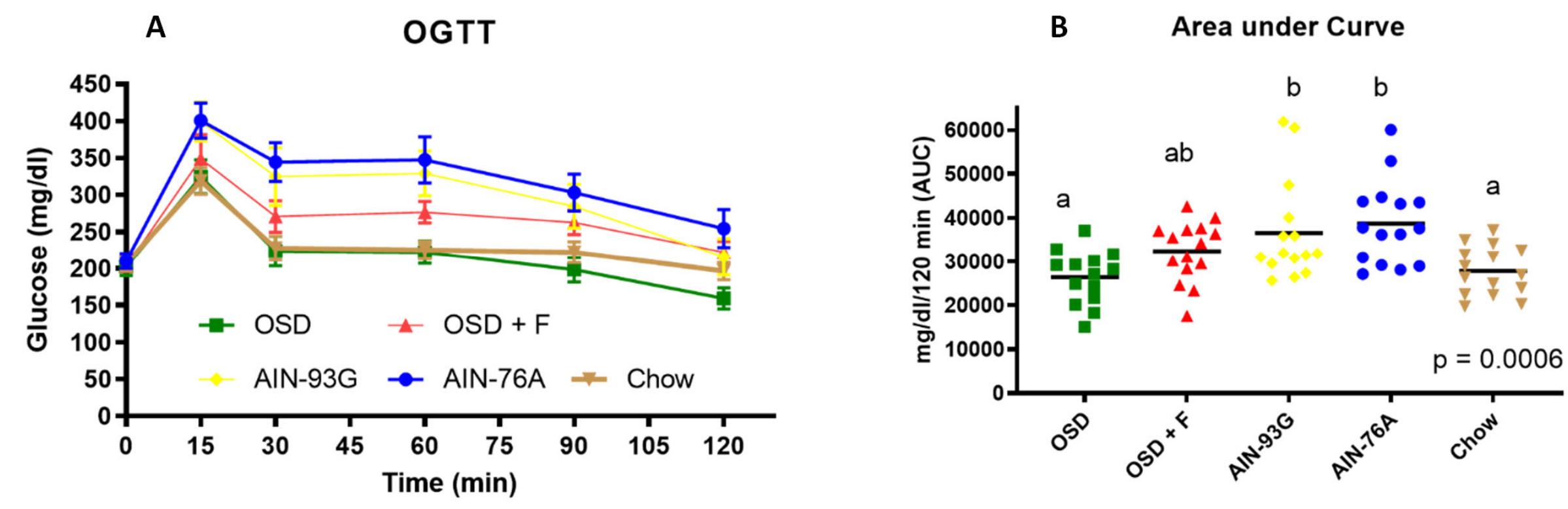

OSD

OSD +

Chow AIN-93G

- AIN-76 
Figure 3. (Study 2). Images of representative cecums and colons for each dietary treatment group after 14 days on either a GBD (5001 or 5002), or high-fiber PDs (100CEL, 100IN, 100FOS, 200CEL, 200IN, 200FOS).

\section{Chows}

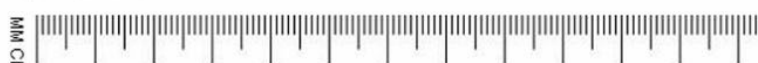
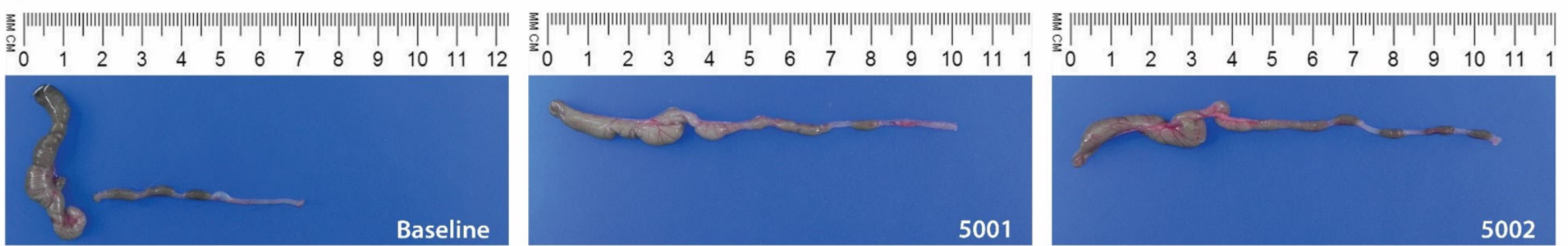

\section{Purified-Ingredient Diets}

三. $\begin{array}{lllllllllllll}0 & 1 & 2 & 3 & 4 & 5 & 6 & 7 & 8 & 9 & 10 & 11 & 1\end{array}$
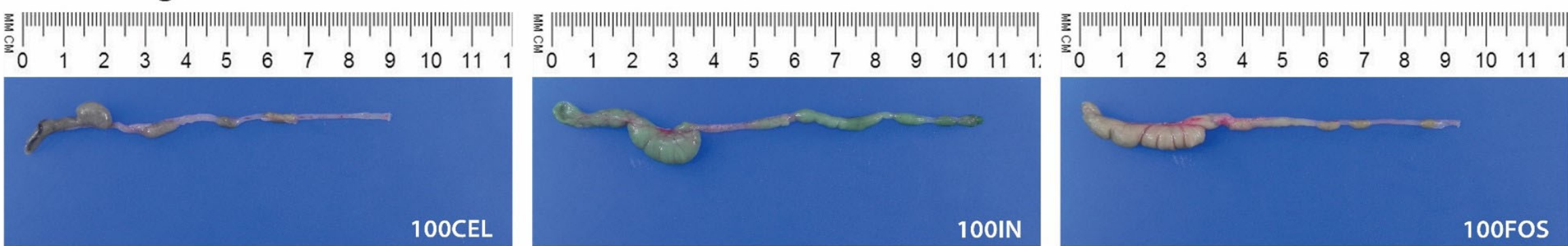

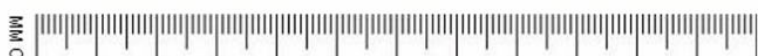

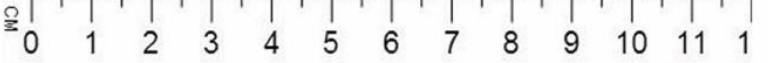

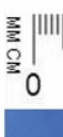

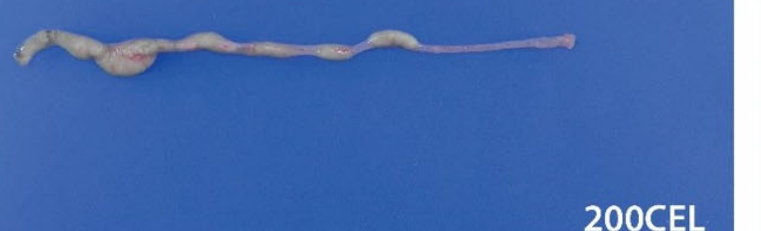

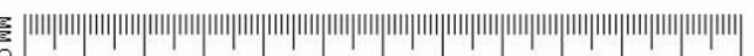

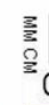

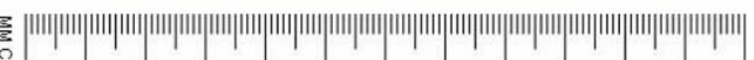
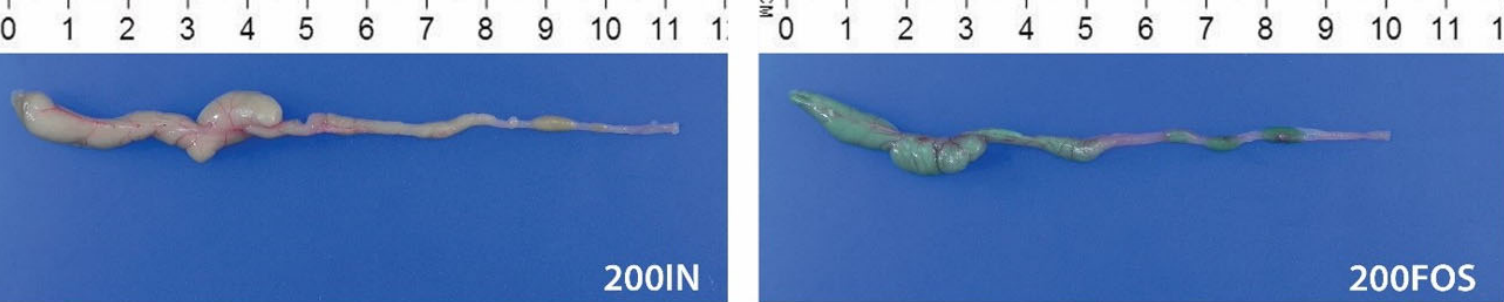
A Bray-Curtis Dissimilarity (ASVs) - Colon Samples

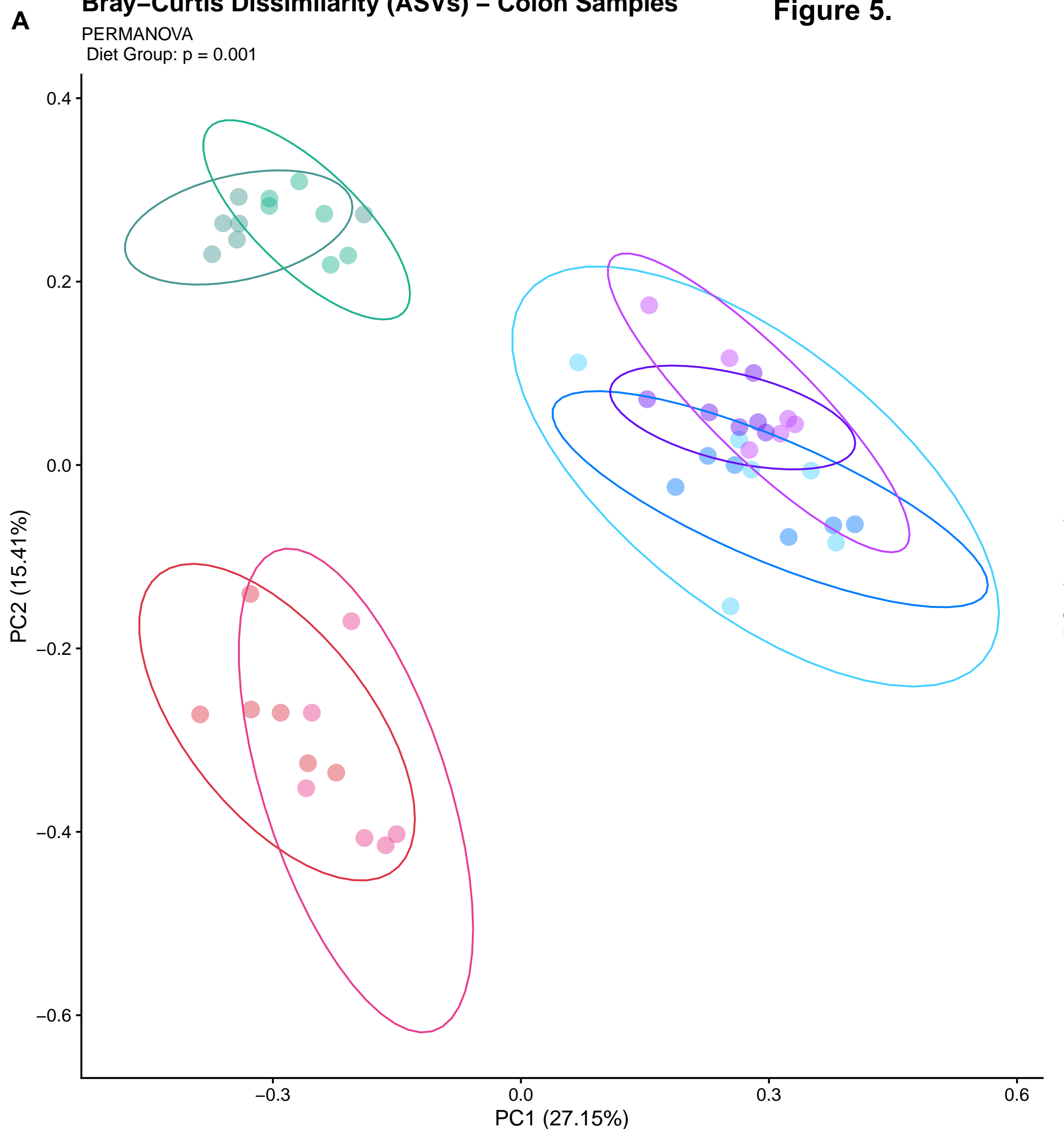

B Bray-Curtis Dissimilarity (ASVs) - Cecum Samples

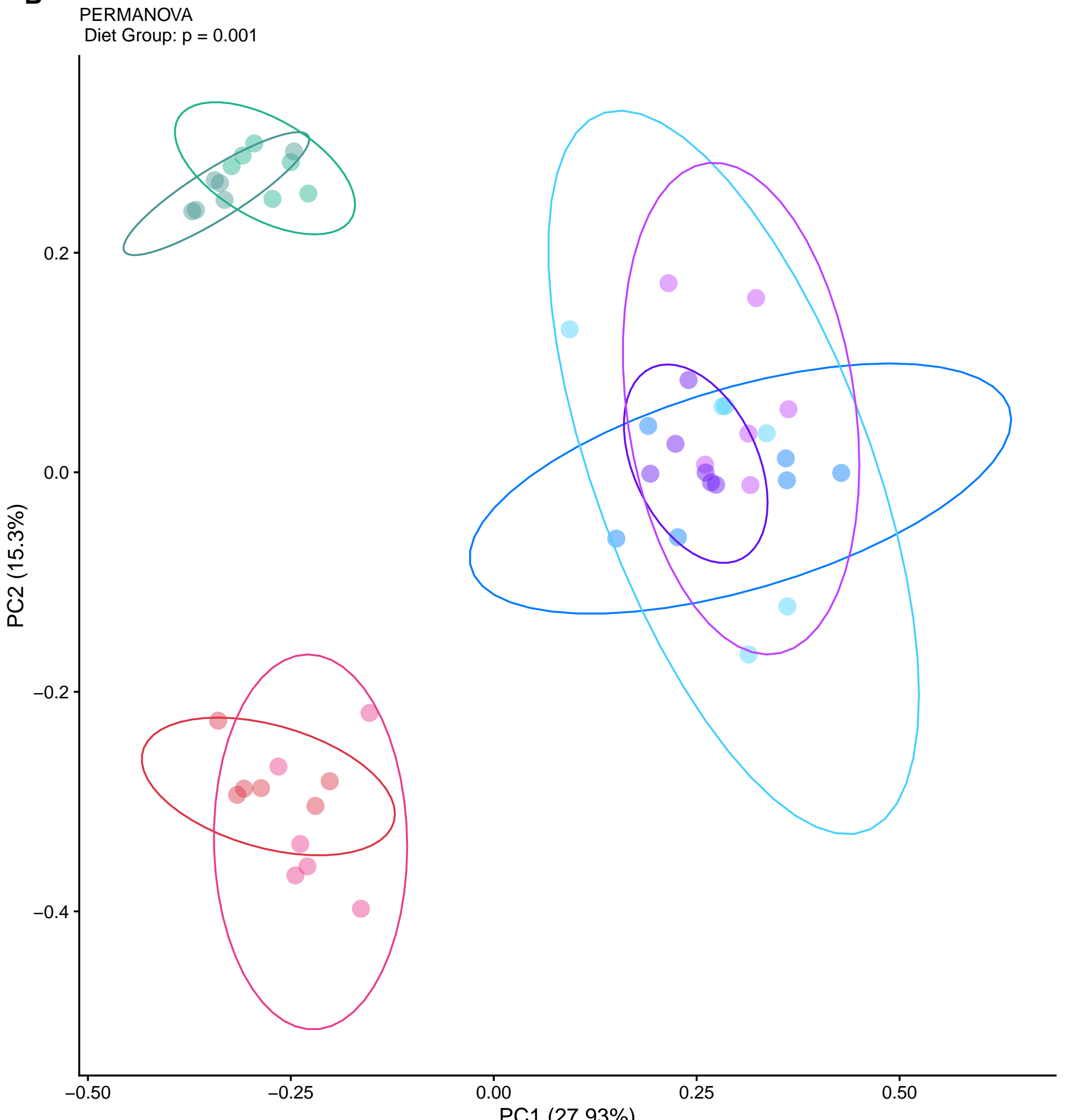

Diet Group

5001

Cellulose_100

Celuose_00

Cellulose_200

Inulin-200

FOS 100

$\ominus$ FOS_100 
Figure 6.

$\mathbf{A}_{1 \infty}$

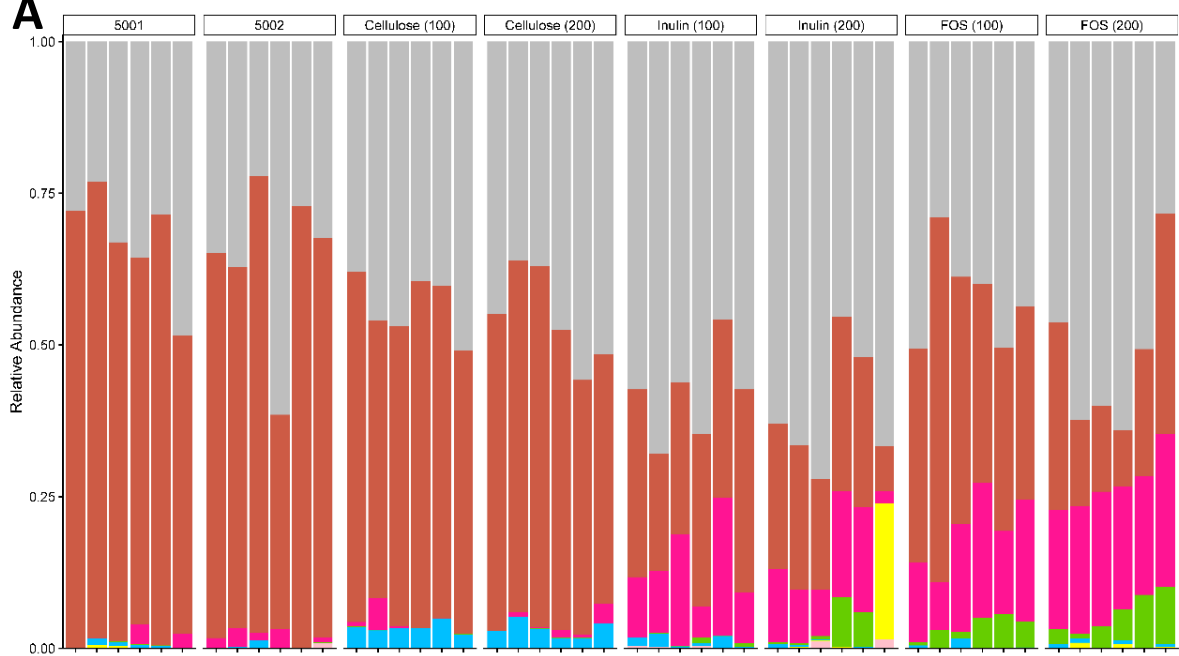

C

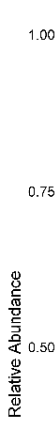

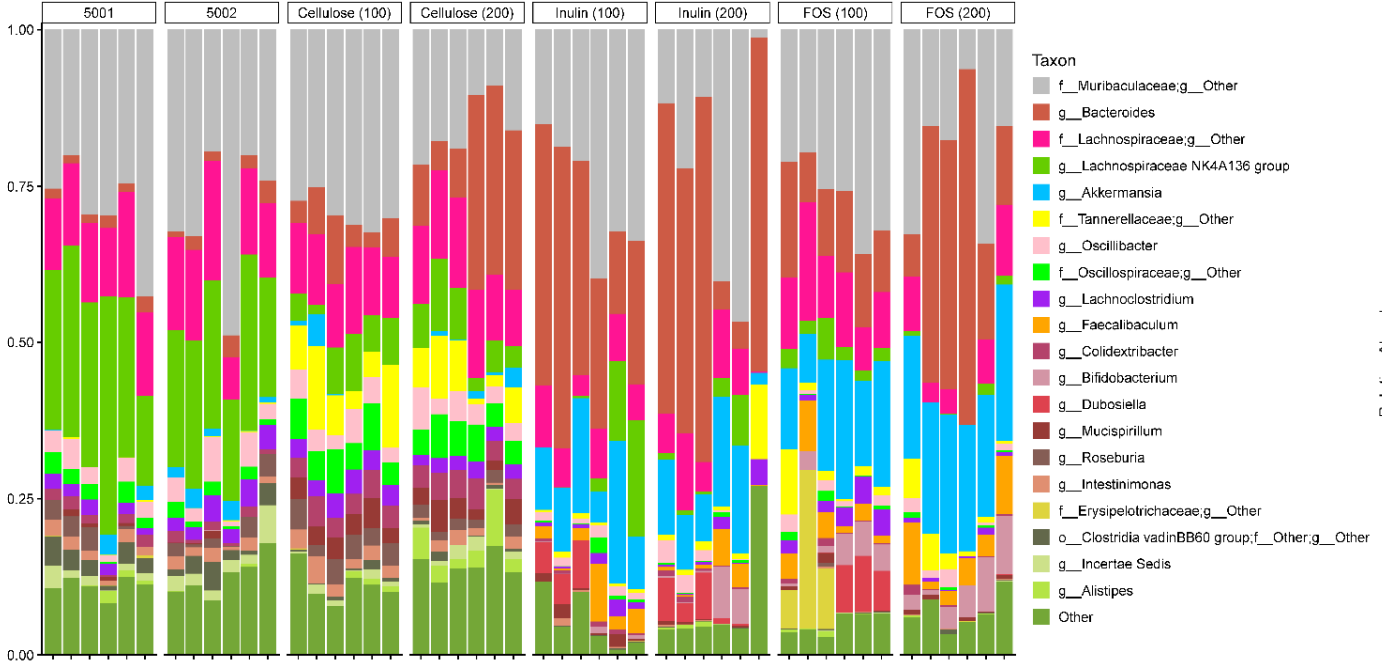

B

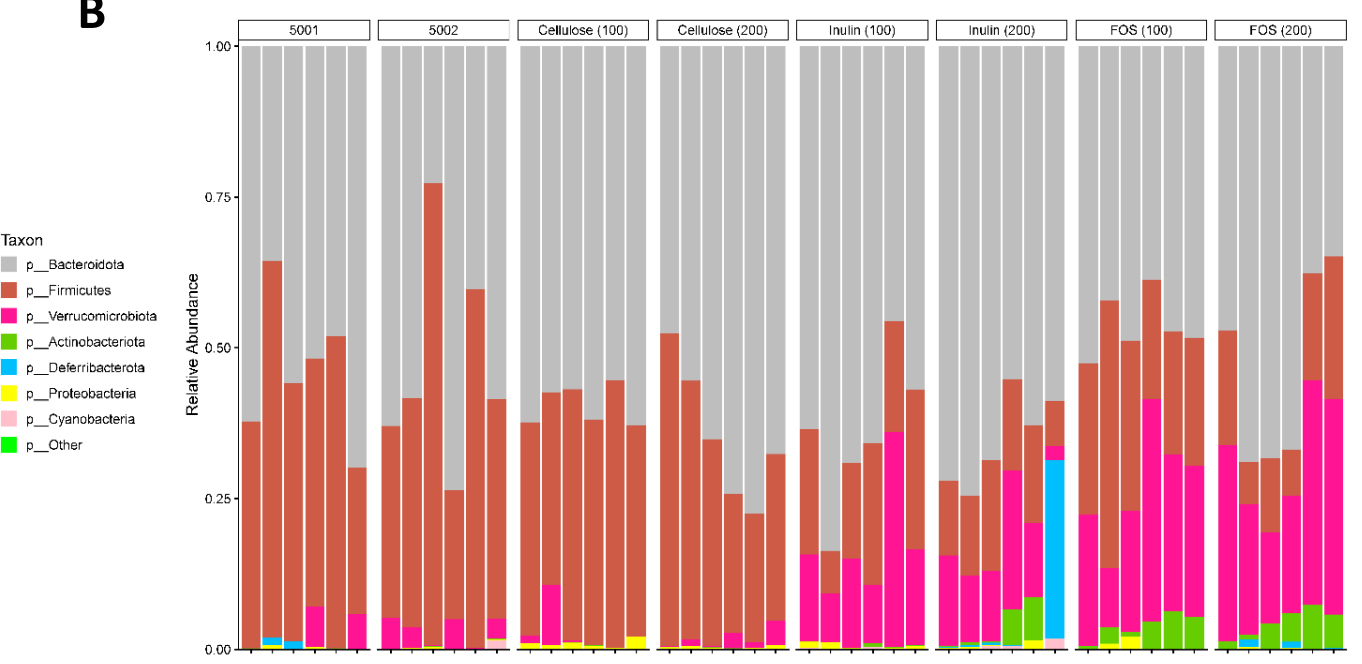

Taxon

1 P_F Fimmunteses 9

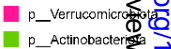

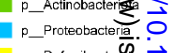

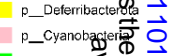

P_other

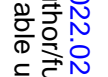

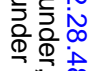

مै

紧哭

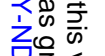

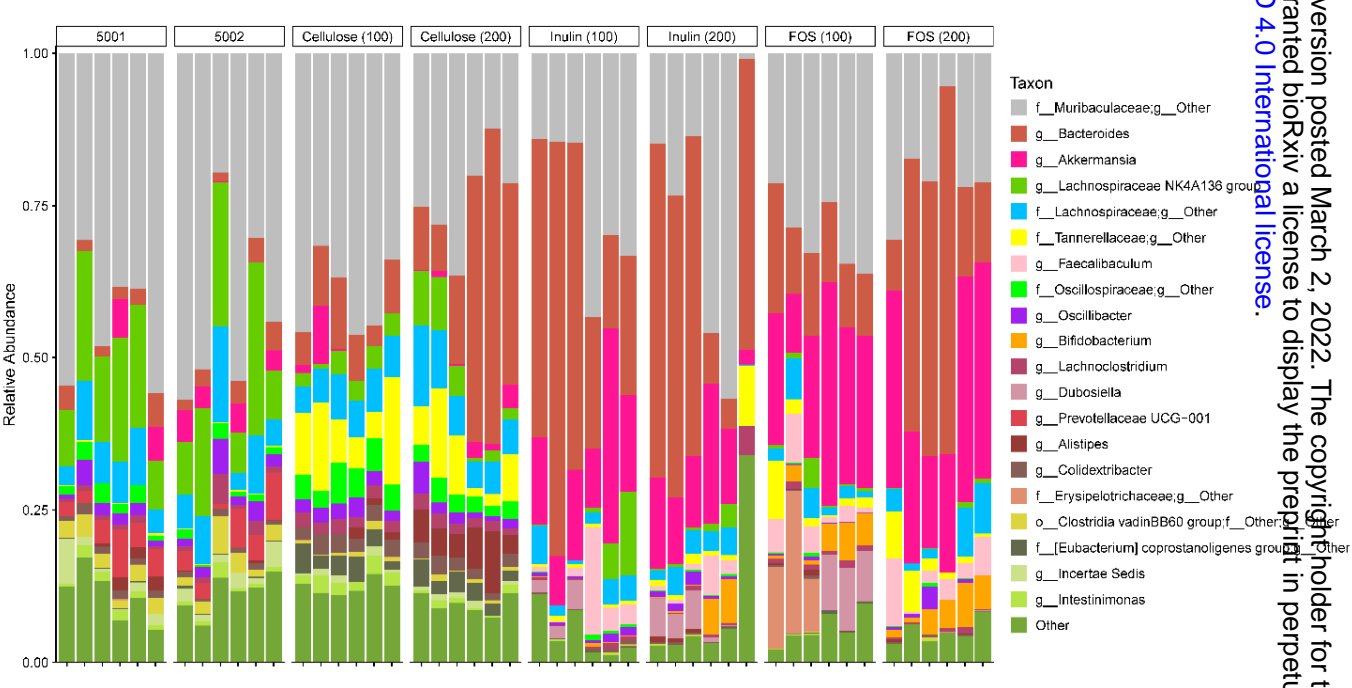

蛋寄.

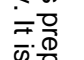

. 
Carbohydrate Degradation and Absorption

ANOVA

Diet Group: $p=0.000302$

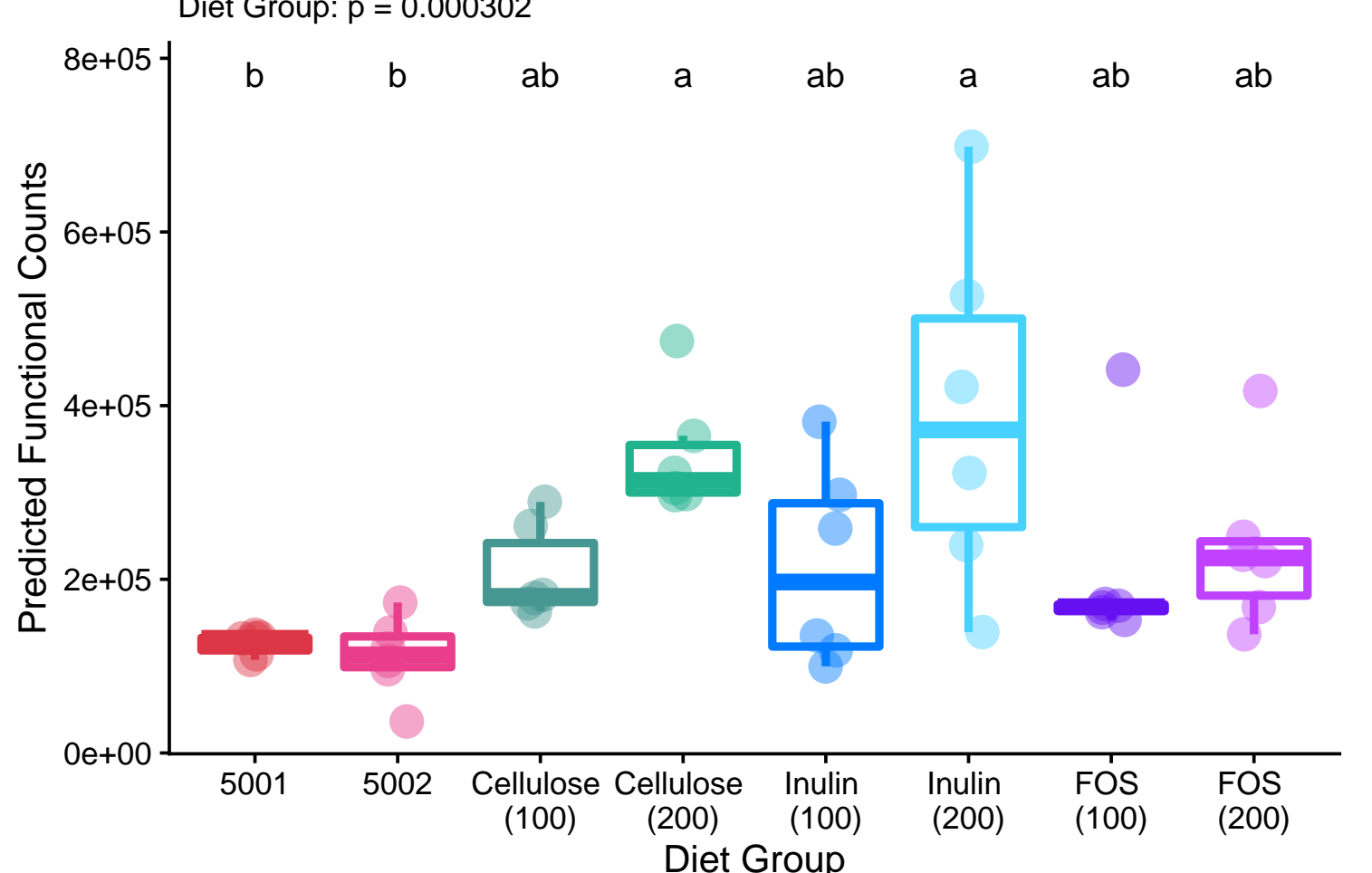

D

Starch and Sucrose Metabolism

ANOVA

ANOVA
Diet Group: $p=1.4 e-05$

C Galactose Metabolism

ANOVA

Figure 7.

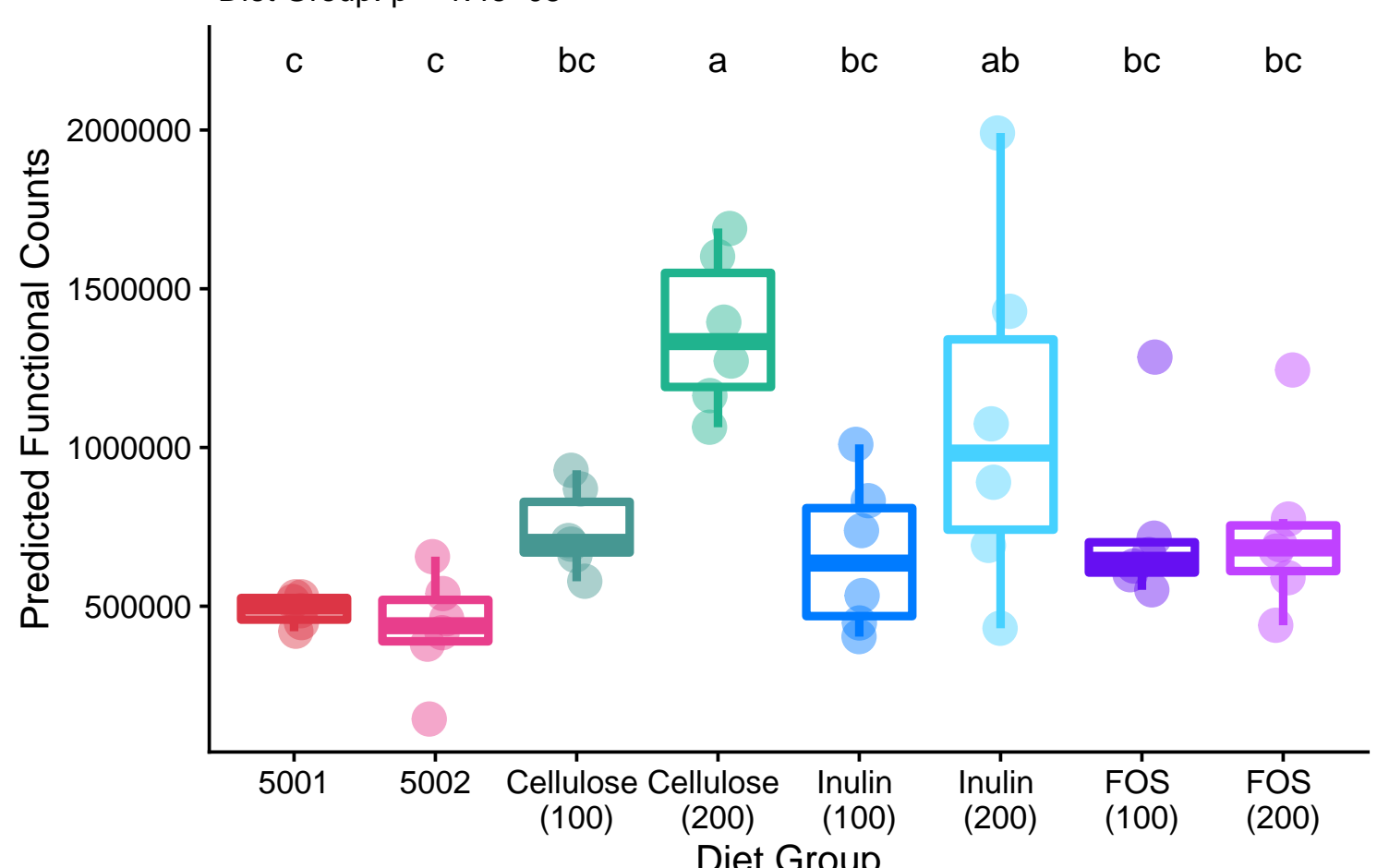

E Fatty Acid Metabolism

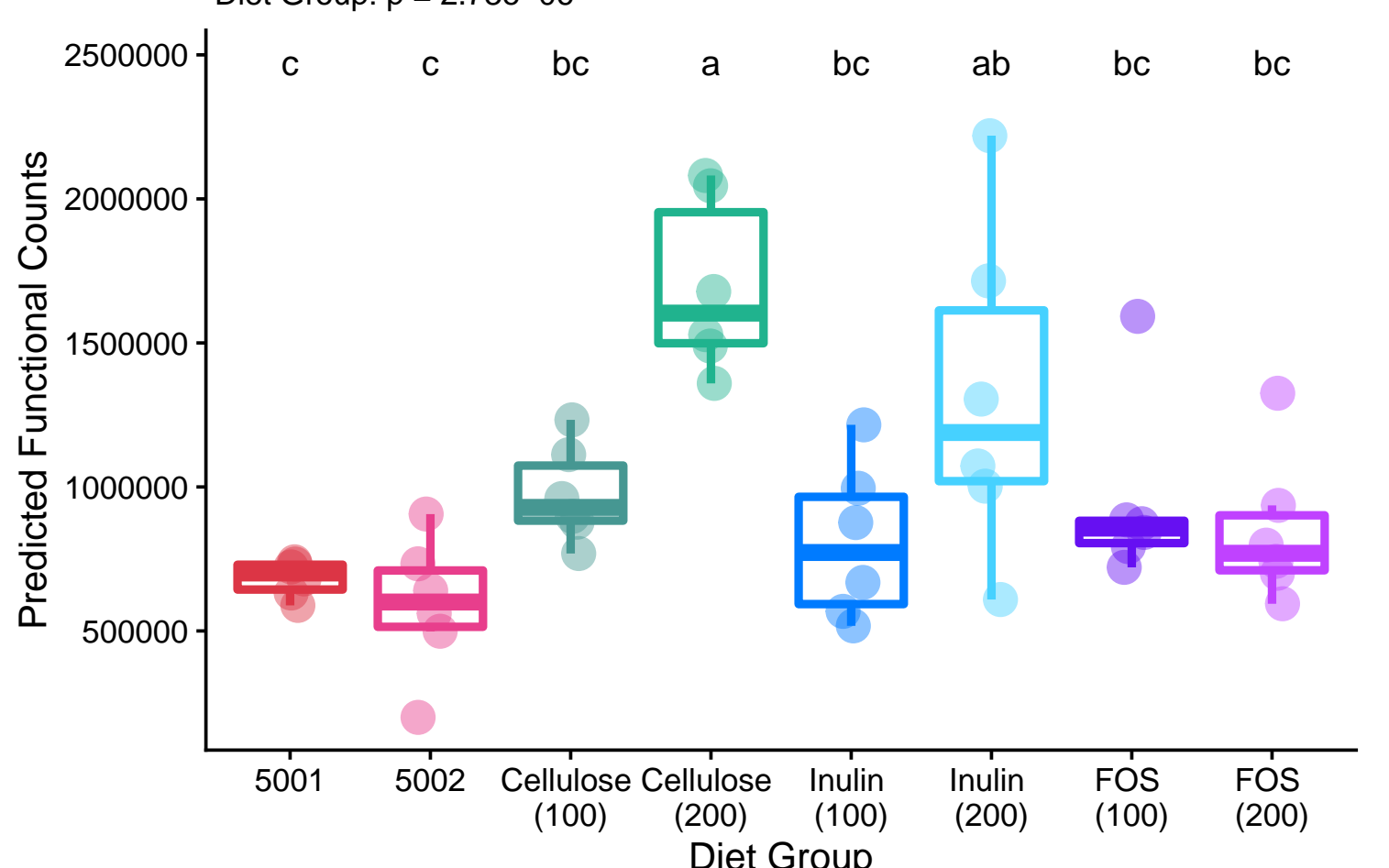
Diet Group
ANOVA

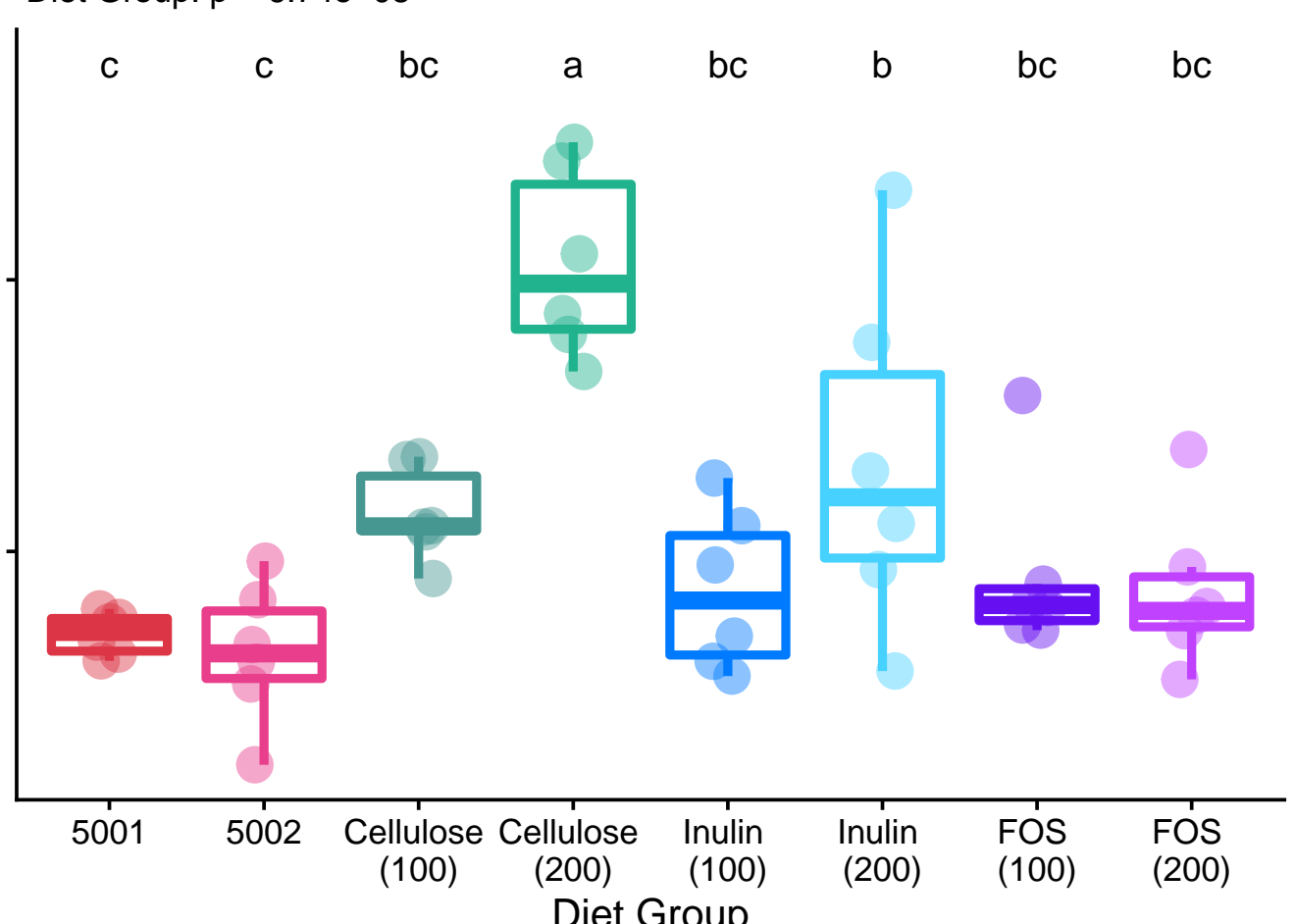

$\mathbf{F}$

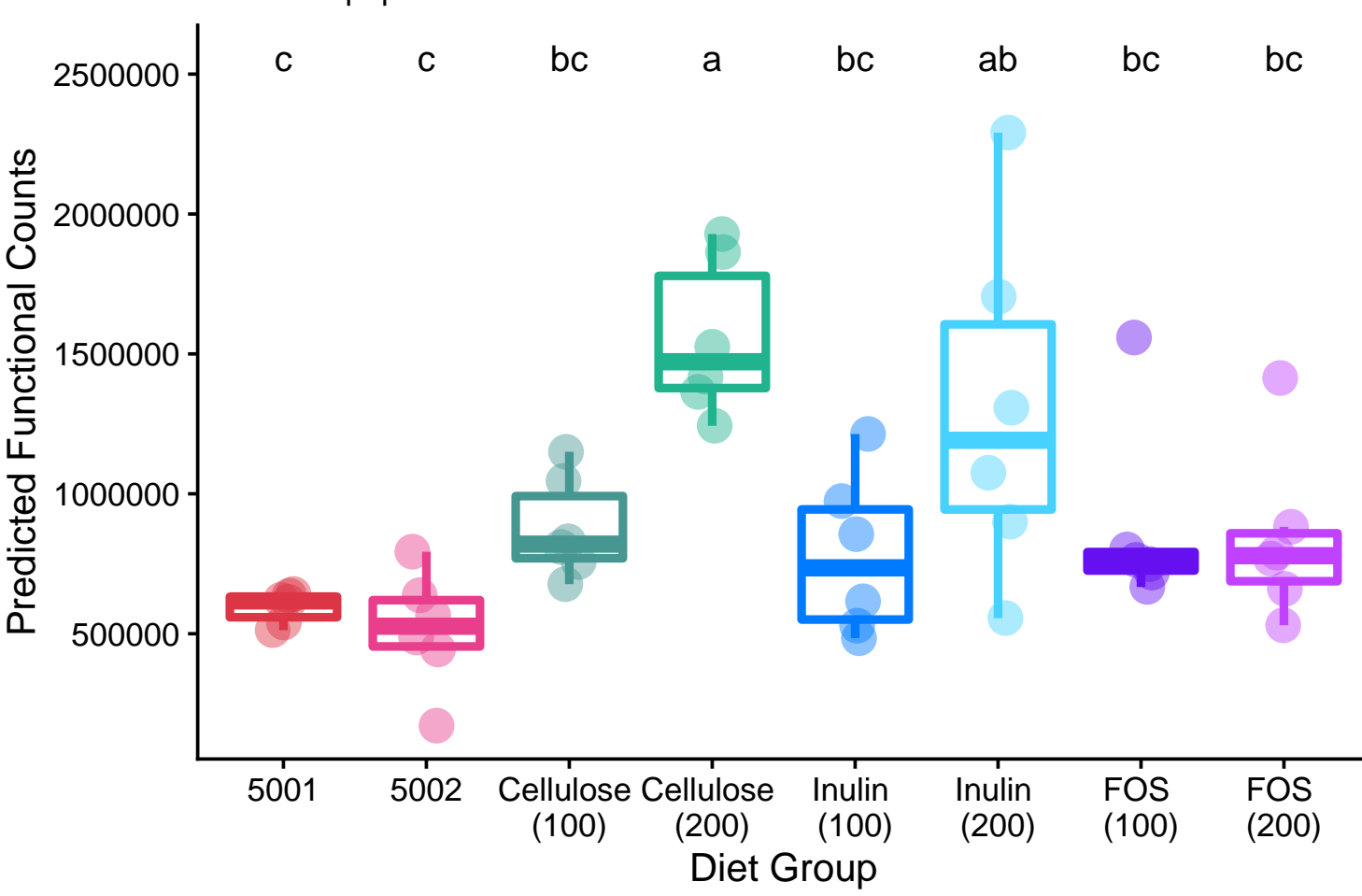

Fatty Acid Biosynthesis

ANOVA
Diet Group: $p=1.91 \mathrm{e}-07$

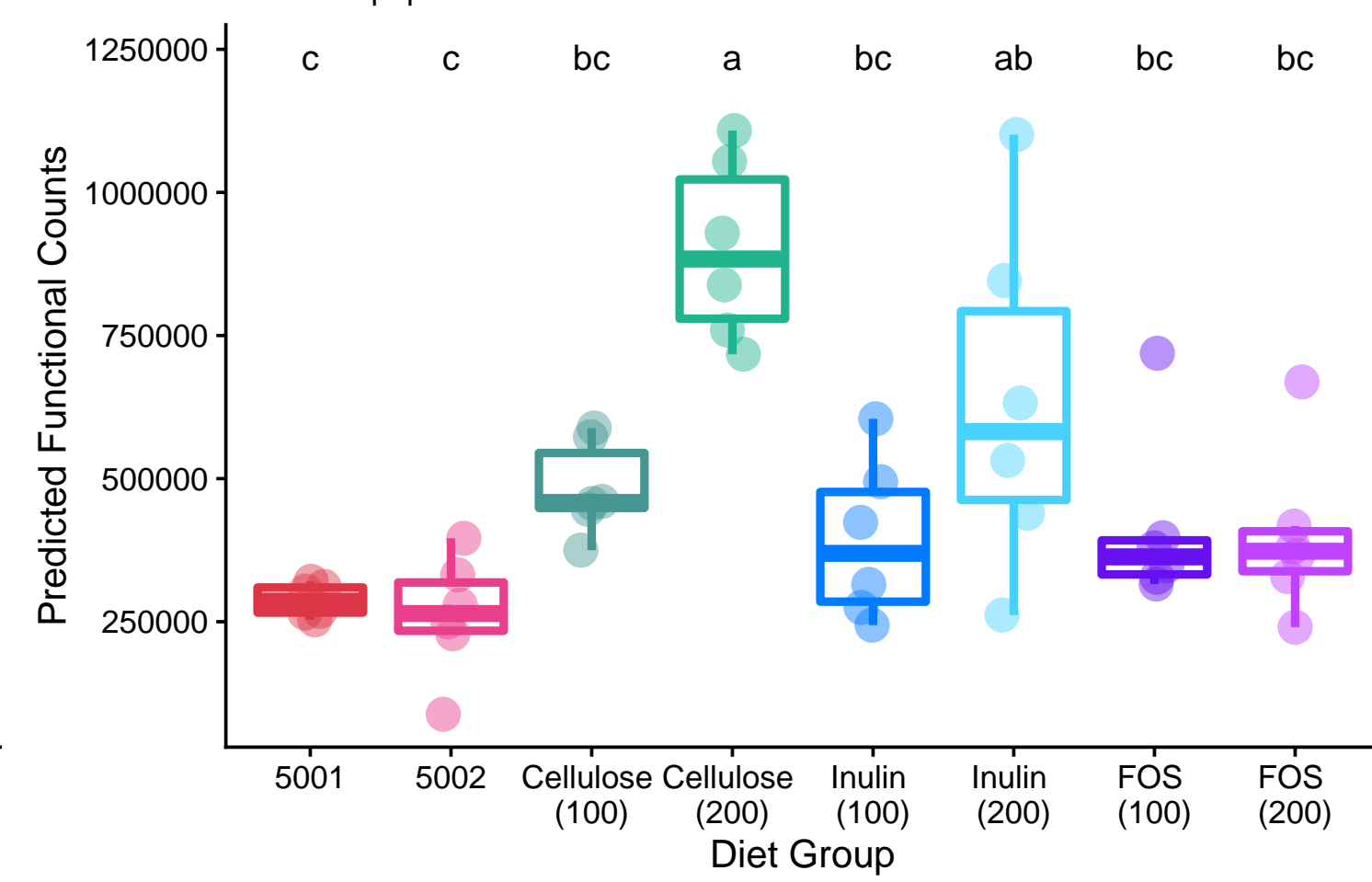

